\title{
An Option Pricing Formula for the GARCH Diffusion Model*
}

\author{
Giovanni Barone-Adesi ${ }^{a}$, Henrik Rasmussen $^{b}$ and Claudia Ravanelli ${ }^{a}$
}

First Version: January 2003

Revised: September 2003

\begin{abstract}
We derive analytically the first four conditional moments of the integrated variance implied by the GARCH diffusion process. From these moments we obtain an analytical closed-form approximation formula to price European options under the GARCH diffusion model. Using Monte Carlo simulations, we show that this approximation formula is accurate for a large set of reasonable parameters. Finally, we use the closed-form option pricing solution to shed light on the qualitative properties of implied volatility surfaces induced by GARCH diffusion models.
\end{abstract}

${ }^{a}$ Institute of Finance, University of Southern Switzerland, Lugano, CH.

${ }^{b}$ Mathematical Institute, University of Oxford, Oxford, UK.

\footnotetext{
${ }^{*}$ Corresponding author: Claudia Ravanelli, University of Southern Switzerland, Institute of Finance, Via
} Buffi 13, CH-6900, Lugano, tel: +41 (0)91 9124786 . E-mail: claudia.ravanelli@lu.unisi.ch. Giovanni Barone-Adesi and Claudia Ravanelli gratefully acknowledge the financial support of the Swiss National Science Foundation and NCCR FINRISK. The authors thank Jerome Detemple and Loriano Mancini for their valuable suggestions. 


\section{Introduction}

In this paper we study European option prices in stochastic volatility models where the underlying asset follows a geometric Brownian motion with instantaneous variance driven by a GARCH diffusion process. Precisely, we derive analytically a closed-form approximation for European option prices under the GARCH diffusion model.

Stochastic volatility models were first introduced by Hull and White (1987), Scott (1987) and Wiggins (1987) to overcome the drawbacks of the Black and Scholes (1973) and Merton (1973) model. Volatilities, stochastically changing over time, account for random behaviours of implied and historical variances and generate some of the log-return features observed in empirical studies $^{1}$. Unfortunately, in the stochastic volatility setting it is difficult to derive closed or analytically tractable option pricing formulas even for European options. The Hull and White (1987) and the Heston (1993) models have an analytical approximation and a quasi-analytical formula to price European options, respectively. For other stochastic volatility models numerical methods are available but these procedures are highly computationally intensive ${ }^{2}$. In this paper, we derive an analytical closed-form approximation for European option prices based on the conditional moments of the integrated variance when the variance is driven by an uncorrelated GARCH diffusion process ${ }^{3}$. Our approximation is very accurate and easy to implement, it can be used to study the implied volatility and the volatility risk premium associated to GARCH diffusion models.

The GARCH diffusion process has several desirable properties. It is positive, mean reverting, with a stationary inverse Gamma distribution and it satisfies the restriction that both historical and implied variances be positive. It also fits the observation that variances seem to be stationary and mean reverting; cf. Scott (1987), Taylor (1994), Jorion (1995) and Guo (1996, 1998). Moreover, the GARCH diffusion model allows for rich pattern behaviours of volatilities and asset prices. For instance, as observed in empirical studies, it produces large autocorrelation in the squared log-returns, arbitrary large kurtosis and finite unconditional moments of log-return distributions up to a given order. By contrast, when the variance follows a square root process as in the Heston (1993) model the corresponding stationary Gamma distribution implies log-return distributions with finite unconditional moments of any order and not very large kurtosis ${ }^{4}$; cf.

\footnotetext{
${ }^{1}$ See , for instance, Mandelbrot (1963) and Fama (1965).

${ }^{2}$ When large trading books have to be quickly and frequently evaluated many procedures are practically not feasible.

${ }^{3}$ This model was first introduced by Wong (1964) and popularized by Nelson (1990).

${ }^{4}$ Rejections of the Heston model are reported by several authors, see for instance Andersen et al. (2002), Jones (2003) and reference therein. Jones (2003, p. 181) found that "the square root stochastic variance model
} 
Genon-Catalot, Jeantheau and Laredo (2000).

Furthermore, Nelson (1990) showed that under the GARCH diffusion model discrete time log-returns follow a $\operatorname{GARCH}(1,1)$ in mean $((\mathrm{GARCH}(1,1)-\mathrm{M})$ process of Engle and Bollerslev (1986)). Hence, the nasty problem of making inference on continuous time parameters may be reduced to the inference on the $\operatorname{GARCH}(1,1)-\mathrm{M}$ model; cf., for instance, Engle and Lee (1996) and Lewis (2000). This is an important advantage over other stochastic volatility models which lack of these simple estimators. In a Monte Carlo study we investigate inference results based on such an estimation procedure. Finally, the GARCH diffusion model is the 'mean reverting' extension of the Hull and White (1987) model where the variance process follows an uncorrelated log-normal process without drift. The GARCH diffusion model makes a marked improvement over the Hull and White model because the mean reverting drift gives stationary variance and log-return processes (cf. Genon-Catalot, Jeantheau and Laredo (2000)) and it can include the volatility risk premium in the variance process. By contrast, for the Hull and White model the analytical option pricing approximation is available only when the drift is equal to zero $^{5}$. Furthermore, the mean reversion of the variance allows to approximate long maturity option prices, while in the Hull and White model the option pricing approximation holds only for short maturity options; cf. Hull and White (1987) and Gesser and Poncet (1997).

Our approximation for option prices under the GARCH diffusion model is based on the Hull and White (1987) formula, which holds when the asset price and the instantaneous variance are uncorrelated. This assumption implies symmetric volatility 'smiles', i.e. symmetric shapes of implied volatilities plotted versus strike prices; cf. Hull and White (1987) and Renault and Touzi (1996). Typically, foreign currency option markets are characterized by symmetric volatility smiles; cf., for instance, Chesney and Scott (1989), Melino and Turbull (1990), Taylor and Xu (1994) and Bollerslev and Zhou (2002). Therefore, the present model can be appropriate to price currency options. Furthermore, also in some index option markets the non zero correlation between price and variance can be neglected without increasing option pricing errors; cf. Chernov and Ghysels (2000) and Jones (2003) for studies on Standard \& Poor's 500 and Standard \& Poor's 100, respectively.

The specific contributions of this paper are the following. We derive analytically the first four conditional moments of the integrated variance implied by the GARCH diffusion process. This result has several important implications. Firstly and foremost, these conditional moments

of Heston is incapable of generating realistic [index] returns behaviour, and data are better represented by a stochastic variance model in the CEV class" as the GARCH diffusion model.

${ }^{5}$ The volatility risk premium seems to be a significant component of the risk premia in many currency markets; cf. Guo (1998) and references therein. 
allow to obtain an analytical closed-form approximation for European option prices under the GARCH diffusion model. This approximation can be easily implemented in any software package (such as Excel spread sheets). Then, just plugging in the model parameters, it provides option prices without any computational efforts. As we will show by Monte Carlo simulations, this approximation is very accurate across different strikes and maturities for a large set of reasonable parameters. Secondly, we propose an analytical approximation for implied volatilities based on the conditional moments of the integrated variance, which allows us to easily study volatility surfaces induced by GARCH diffusion models. Thirdly, the conditional moments of the integrated variance implied by the GARCH diffusion process generalize the conditional moments derived by Hull and White (1987) for log-normal variance processes. Finally, the conditional moments of the integrated variance can be used to estimate the continuous time parameters of the GARCH diffusion model using high frequency data ${ }^{6}$. As already mentioned, Nelson's theory suggest an appealing estimation procedure for the GARCH diffusion model parameters. By Monte Carlo simulation we investigate the accuracy of such inference results.

The rest of the paper is organized as follows. Section 2 introduces the GARCH diffusion model. Section 3 presents the analytical approximation formula to price European vanilla options under the GARCH diffusion model. In Section 4, using Monte Carlo simulations, the accuracy of the approximation is investigated across different strike prices and time to maturities for different parameter choices. Section 5 studies implied volatility surfaces induced by the model. Section 6 studies the accuracy of the inference on the GARCH diffusion model based on the Nelson's theory and Section 7 concludes.

\section{The Model}

Let $S=\left(S_{t}\right)_{t \geq 0}$ be the underlying currency price and $V=\left(V_{t}\right)_{t \geq 0}$ its latent instantaneous variance. We assume that $\left(S_{t}, V_{t}\right)_{t \geq 0}$ satisfies the two-dimensional GARCH diffusion model

$$
\begin{aligned}
& d S_{t}=\mu S_{t} d t+\sqrt{V_{t}} S_{t} d B_{t}, \\
& d V_{t}=\left(c_{1}-c_{2} V_{t}\right) d t+c_{3} V_{t} d W_{t},
\end{aligned}
$$

where $c_{1}, c_{2}$ and $c_{3}$ are positive constants, $\mu$ is the positive constant drift of $d S_{t} / S_{t}, B$ and $W$ are mutually independent one-dimensional Brownian motions on some filtered probability space $\left(\Omega, \mathcal{F}, \mathcal{F}_{t}, \mathbb{P}\right)$ and $\mathbb{P}$ is the objective measure. We set the initial time $t=0$ and $\left(S_{0}, V_{0}\right) \in \mathbb{R}^{+} \times \mathbb{R}^{+}$.

\footnotetext{
${ }^{6}$ By matching the sample moments of the realized volatility with the conditional moments of the integrated variance one has a standard and easy to compute GMM-type estimator for the underlying model parameters; cf. Bollerslev and Zhou (2002).
} 
The $V$ process is mean reverting, $c_{1} / c_{2}$ determines the run mean value and $c_{2}$ is the reversion rate (see also equation (4)). For 'small' $c_{2}$ the mean reversion is 'weak' and $V_{t}$ tends to stay above (or below) the run mean value for long periods, i.e. to volatility cluster. The parameter $c_{3}$ determines the random behaviour of the volatility: for $c_{3}=0$ the volatility process is deterministic, for $c_{3}>0$ the kurtosis of log-return distributions is larger than 3 . When $c_{1}=c_{2}=0$, the GARCH diffusion process reduces to the log-normal process without drift in the Hull and White (1987) model.

Given $V_{0}>0, V_{t}$ is positive $\mathbb{P}$-almost surely $\forall t \geq 0$, and the strong solution is

$$
V_{t}=V_{0} e^{-\left(c_{2}+\frac{1}{2} c_{3}^{2}\right) t+c_{3} W_{t}}+c_{1} \int_{0}^{t} e^{\left(c_{2}+\frac{1}{2} c_{3}^{2}\right)(s-t)+c_{3}\left(W_{t}-W_{s}\right)} d s
$$

see Karatzas and Shreve (1991), p. 360. The stationary distribution of $V$ is the Inverse Gamma distribution (cf. Nelson (1990)) with parameters $1+2 c_{2} / c_{3}^{2}$ and $c_{3}^{2} / 2 c_{1}$, i.e. $1 / V_{t} \leadsto \Gamma(1+$ $\left.2 c_{2} / c_{3}^{2}, c_{3}^{2} / 2 c_{1}\right)$. Hence $V_{t}$ has finite moments up to order $r$ if and only if $r<1+2 c_{2} / c_{3}^{2}$. This implies that log-return distributions have finite unconditional moments up to order $2 r$. Empirical studies showed that log-return distributions have finite moments up to some given order. Moreover, when $c_{3}^{2} \rightarrow 2 c_{2}^{+}$, the kurtosis of log-return distributions tends to infinity and the correlation between squared log-returns approaches to $1 / 3$.

When $2 c_{2}>c_{3}^{2}$, the $V$ process is strictly stationary, ergodic with conditional mean and variance

$$
\begin{aligned}
\mathbb{E}\left[V_{t} \mid V_{0}\right]=\frac{c_{1}}{c_{2}}+ & \left(V_{0}-\frac{c_{1}}{c_{2}}\right) e^{-c_{2} t} \\
\operatorname{Var}\left[V_{t} \mid V_{0}\right]= & \frac{\left(c_{1} / c_{2}\right)^{2}}{2 c_{2} / c_{3}^{2}-1}+e^{-c_{2} t} \frac{2\left(c_{1} / c_{2}\right)\left(V_{0}-\left(c_{1} / c_{2}\right)\right)}{c_{2} / c_{3}^{2}-1}-e^{-2 c_{2} t}\left(V_{0}-\left(c_{1} / c_{2}\right)\right)^{2} \\
& +e^{\left(c_{3}^{2}-2 c_{2}\right) t}\left(V_{0}^{2}-\frac{2 V_{0}\left(c_{1} / c_{2}\right)}{1-c_{3}^{2} / c_{2}}+\frac{\left(c_{1} / c_{2}\right)^{2}}{\left(1-c_{3}^{2} / 2 c_{2}\right)\left(1-c_{3}^{2} / c_{2}\right)}\right) .
\end{aligned}
$$

The unconditional expectation of (4) and (5) give the unconditional mean and variance of $\mathrm{V}$

$$
\mathbb{E}\left[V_{1}\right]=\frac{c_{1}}{c_{2}}, \quad \operatorname{Var}\left[V_{1}\right]=\frac{\left(c_{1} / c_{2}\right)^{2}}{2 c_{2} / c_{3}^{2}-1} .
$$

In Section 4 we will infer some reasonable parameters for the variance process using equations (6). Higher order unconditional moments of $V$ can be derived by the stationary Inverse Gamma distribution.

In the following section we will derive an analytical approximation formula for European options when the underlying currency price satisfies equations (1)-(2). 


\section{The Option Pricing Formula}

Given the model (1)-(2), a foreign currency option price $f(S, V, t)$ satisfies the following partial differential equation

$$
\frac{1}{2} V S^{2} \frac{\partial f^{2}}{\partial S^{2}}+\frac{1}{2} c_{3}^{2} V^{2} \frac{\partial f^{2}}{\partial V^{2}}+\left(r_{d}-r_{f}\right) S \frac{\partial f}{\partial S}+\left(\left(c_{1}-c_{2}\right) V-\lambda(S, V, t)\right) \frac{\partial f}{\partial V}-\left(r_{d}-r_{f}\right) f+\frac{\partial f}{\partial t}=0,
$$

where $r_{d}$ and $r_{f}$ are the domestic and the foreign interest rates, respectively, and the unspecified term $\lambda(S, V, t)$ represents the market price of risk associated to the variance $V$. As in other studies (see, for instance, Chesney and Scott (1989), Heston (1993) and Jones (2003)), we specify the volatility risk premium as $\lambda(V, S, t)=\lambda V$. The risk-adjusted process still remains a GARCH diffusion process,

$$
\begin{aligned}
& d S_{t}=\left(r_{d}-r_{f}\right) S_{t} d t+\sqrt{V_{t}} S_{t} d B_{t}^{*}, \\
& d V_{t}=\left(c_{1}-c_{2}^{*} V_{t}\right) d t+c_{3} V_{t} d W_{t}^{*},
\end{aligned}
$$

where $c_{2}^{*}=c_{2}+\lambda, B^{*}$ and $W^{*}$ are mutually independent Brownian motions under the riskadjusted measure $\mathbb{P}^{*}$.

For the risk-adjusted dynamics in equations (7)-(8) the option pricing result in Hull and White (1987) holds: the fair price value $C_{s v}$ for a European call with time to maturity $T$ and strike price $K$ is given by

$$
C_{s v}=\int_{0}^{\infty} C_{b s}\left(\bar{V}_{T}\right) f\left(\bar{V}_{T} \mid V_{0}\right) d \bar{V}_{T}
$$

where $C_{b s}$ is the Black and Scholes (1973) option price, $\bar{V}_{T}$ is the integrated variance over the time to maturity $T$, i.e.

$$
\bar{V}_{T}:=\frac{1}{T} \int_{0}^{T} V_{t} d t
$$

and $f\left(\bar{V}_{T} \mid V_{0}\right)$ is the conditional density function of $\bar{V}_{T}$ given $V_{0}$. The integrated variance density $f\left(\bar{V}_{T} \mid V_{0}\right)$ is not known and the option price $C_{s v}$ is not available in closed-form. The expectation in equation (9) can be computed by Monte Carlo simulation but such a procedure is very time-consuming. Hull and White (1987) provided an analytical approximation for $C_{s v}$ in (9). Precisely, they computed the Taylor expansion of $C_{b s}$ in equation (9) around the conditional mean of $\bar{V}_{T}$ obtaining a series option pricing formula that involves only the conditional moments of $\bar{V}_{T}$ and the sensitivities of the Black and Scholes price to the variance. Denoting by $M_{1}:=$ $\mathbb{E}\left[\bar{V}_{T} \mid V_{0}\right]$ the conditional mean of $\bar{V}_{T}$ and $M_{i c}:=\mathbb{E}\left[\left(\bar{V}_{T}-M_{1}\right)^{i} \mid V_{0}\right] \quad i \geq 2$ the i-th centered conditional moment of $\bar{V}_{T}$, the option pricing series is

$$
C_{s v}=C_{b s}\left(M_{1}\right)+\left.\frac{1}{2} M_{2 c} \frac{\partial^{2} C_{b s}}{\partial \bar{V}_{T}^{2}}\right|_{\bar{V}_{T}=M_{1}}+\left.\frac{1}{6} M_{3 c} \frac{\partial^{3} C_{b s}}{\partial \bar{V}_{T}^{3}}\right|_{\bar{V}_{T}=M_{1}}+\left.\frac{1}{24} M_{4 c} \frac{\partial^{4} C_{b s}}{\partial \bar{V}_{T}^{4}}\right|_{\bar{V}_{T}=M_{1}}+\ldots
$$


where the derivatives are

$$
\begin{aligned}
\frac{\partial C_{b s}}{\partial \bar{V}_{T}}= & \frac{e^{-r_{f} T} S_{0} \sqrt{T} e^{-d_{1}^{2} / 2}}{\sqrt{8 \pi \bar{V}_{T}}}, \\
\frac{\partial^{2} C_{b s}}{\partial \bar{V}_{T}^{2}}= & \frac{\partial C_{b s}}{\partial \bar{V}_{T}}\left[\frac{1}{2} \frac{m^{2}}{\left(\bar{V}_{T} T\right)^{2}}-\frac{1}{2 \bar{V}_{T} T}-\frac{1}{8}\right] T, \\
\frac{\partial^{3} C_{b s}}{\partial \bar{V}_{T}^{3}}= & \frac{\partial C_{b s}}{\partial \bar{V}_{T}}\left[\frac{m^{4}}{4\left(\bar{V}_{T}^{T}\right)^{4}}-\frac{m^{2}\left(12+\bar{V}_{T} T\right)}{8\left(\bar{V}_{T} T\right)^{3}}+\frac{48+8 \bar{V}_{T} T+\left(\bar{V}_{T} T\right)^{2}}{64\left(\bar{V}_{T} T\right)^{2}}\right] T^{2}, \\
\frac{\partial^{4} C_{b s}}{\partial \bar{V}_{T}^{4}}= & \frac{\partial C_{b s}}{\partial \bar{V}_{T}}\left[\frac{1}{8} \frac{m^{6}}{\left(\bar{V}_{T} T\right)^{6}}-\frac{3}{32} \frac{m^{4}\left(20+\bar{V}_{T} T\right)}{\left(\bar{V}_{T} T\right)^{5}}+\frac{3}{128} \frac{m^{2}\left(240+24 \bar{V}_{T} T+\left(\bar{V}_{T} T\right)^{2}\right)}{\left(\bar{V}_{T} T\right)^{4}}\right. \\
& \left.\quad-\frac{\left(960+144 \bar{V}_{T} T+12\left(\bar{V}_{T} T\right)^{2}+\left(\bar{V}_{T} T\right)^{3}\right)}{512\left(\bar{V}_{T} T\right)^{3}}\right] T^{3},
\end{aligned}
$$

and $m:=\log \left(S_{0} / K\right)+\left(r_{d}-r_{f}\right) T$. So far, the conditional moments of the integrated variance have been calculated analytically only for few specifications of the variance process

1. for the mean reverting Ornstein-Uhlenbeck process ${ }^{7}$ Cox and Miller (1972, Sec. 5.8) derived the first two conditional moments of $\bar{V}_{T}$;

2. for the geometric Brownian motion with drift Hull and White (1987) derived the first two conditional moments of $\bar{V}_{T}$ and the first three conditional moments of $\bar{V}_{T}$ for the variance process without drift;

3. for the squared root process Bollerslev and Zhou (2002) derived the first two conditional moments. Lewis (2000a) derived the first four conditional moments of the integrated variance for the general class of affine processes (including the squared root process).

Given the analytical conditional moments of $\bar{V}_{T}$ it is very easy to price European options by the series approximation (11). Garcia, Lewis and Renault (2001) use this formula to price European options under the Heston model notwithstanding the Heston option pricing formula; cf. also Ball and Roma (1994). Indeed, implementing integral solutions for option prices, such as the Heston formula, can be very delicate due to divergence of the integrand in some regions of the parameter space.

We derive the first four conditional moments of $\bar{V}_{T}$ when the variance $V$ is driven by the GARCH diffusion process (2). The first conditional moment is already known in the literature. The second, the third and the fourth are believed to be new. Higher order moments are essential to capture the 'smile' effect of implied volatilities; cf., for instance, Bodurtha and

\footnotetext{
${ }^{7}$ We recall that the mean reverting Ornstein-Uhlenbeck process is normally distributed and then can not ensure positive variance.
} 
Courtadon (1987) for PHLX foreign currency options and Lewis (2000). We denote these conditional moments by $M_{1}^{g d}, M_{2 c}^{g d}, M_{3 c}^{g d}$ and $M_{4 c}^{g d}$. Here we state $M_{1}^{g d}, M_{2 c}^{g d}$ and the calculations are given in Appendix A. The third and the fourth conditional moments are more involved and are available from the authors on request.

Proposition 3.1 Let $V=\left(V_{t}\right)_{t \geq 0}$ to satisfy the stochastic differential equation (2). Given $\left(V_{0}, c_{1}\right) \in \mathbb{R}^{+} \times \mathbb{R}^{+}$and $c_{2}>c_{3}^{2}$, the first and the second conditional moment of the integrated variance $\bar{V}_{T}$ are

$$
\begin{aligned}
M_{1}^{g d} & :=\mathbb{E}\left[\bar{V}_{T} \mid V_{0}\right]=\frac{c_{1}}{c_{2}}+\left(V_{0}-\frac{c_{1}}{c_{2}}\right) \frac{1-e^{-c_{2} T}}{c_{2} T}, \\
M_{2 c}^{g d} & :=\mathbb{E}\left[\left(\bar{V}_{T}-M_{1}^{g d}\right)^{2} \mid V_{0}\right]=-\frac{e^{-2 T c_{2}}\left(c_{2} V_{0}-c_{1}\right)^{2}}{T^{2} c_{2}^{4}} \\
& +\frac{2 e^{\left(c_{3}^{2}-2 c_{2}\right) T}\left(2 c_{1}^{2}+2 c_{1}\left(c_{3}^{2}-2 c_{2}\right) V_{0}+\left(2 c_{2}^{2}-3 c_{2} c_{3}^{2}+c_{3}^{4}\right) V_{0}^{2}\right)}{T^{2}\left(c_{2}-c_{3}^{2}\right)^{2}\left(2 c_{2}-c_{3}^{2}\right)^{2}} \\
& -\frac{c_{3}^{2}\left(c_{1}^{2}\left(4 c_{2}\left(3-T c_{2}\right)+\left(2 T c_{2}-5\right) c_{3}^{2}\right)+2 c_{1} c_{2}\left(-2 c_{2}+c_{3}^{2}\right) V_{0}+c_{2}^{2}\left(-2 c_{2}+c_{3}^{2}\right) V_{0}^{2}\right)}{T^{2} c_{2}^{4}\left(-2 c_{2}+c_{3}^{2}\right)^{2}} \\
& +\frac{2 e^{-T c_{2}} c_{3}^{2}\left(2 c_{1}^{2}\left(T c_{2}^{2}-\left(1+T c_{2}\right) c_{3}^{2}\right)+2 c_{1} c_{2}^{2}\left(1-T c_{2}+T c_{3}^{2}\right) V_{0}+c_{2}^{2}\left(c_{3}^{2}-c_{2}\right) V_{0}^{2}\right)}{T^{2} c_{2}^{4}\left(c_{2}-c_{3}^{2}\right)^{2}} .
\end{aligned}
$$

These moments are obtained using properties of Brownian motion such as independence and stationarity of non-overlapping increments and the linearity of $d V_{t}$ in $V_{t}$. As already observed, for $c_{1}=0$ the GARCH diffusion process reduces to the log-normal process with drift and then $M_{1}^{g d}, M_{2 c}^{g d}$ reduce to the conditional mean and variance of $\bar{V}_{T}$ in Hull and White (1987), p. 287.

Given the first four conditional moments of $\bar{V}_{T}$, under the GARCH diffusion model the call price is

$$
\widetilde{C}^{g d}=C_{b s}\left(M_{1}^{g d}\right)+\left.\frac{1}{2} M_{2 c}^{g d} \frac{\partial^{2} C_{b s}}{\partial \bar{V}_{T}^{2}}\right|_{\bar{V}_{T}=M_{1}^{g d}}+\left.\frac{1}{6} M_{3 c}^{g d} \frac{\partial^{3} C_{b s}}{\partial \bar{V}_{T}^{3}}\right|_{\bar{V}_{T}=M_{1}^{g d}}+\left.\frac{1}{24} M_{4 c}^{g d} \frac{\partial^{4} C_{b s}}{\partial \bar{V}_{T}^{4}}\right|_{\bar{V}_{T}=M_{1}^{g d}} .
$$

Although $M_{1}^{g d}, M_{2 c}^{g d}, M_{3 c}^{g d}$ and $M_{4 c}^{g d}$ are rather nasty, the closed-form approximation formula (15) can be easily implemented in any software package (such as Excel spread sheets) providing option prices by just plugging in model parameters without any computational efforts.

As we will show in the next section, our approximation formula (15) is very accurate for a large set of reasonable parameters. Intuitively, when the time to maturity $T$ is 'short', $\bar{V}_{T}$ is not too far from $M_{1}^{g d}:=\mathbb{E}\left[\bar{V}_{T} \mid V_{0}\right]$, then we expect approximation (15) to converge quickly. 
When the time to maturity $T$ increases, $M_{1}^{g d}$ tends to the run mean value of $\mathrm{V}, \mathbb{E}\left[M_{1}^{g d}\right]=c_{1} / c_{2}$, and $M_{2 c}, M_{3 c}$ and $M_{4 c}$ go to zero. Therefore, we expect the approximation formula (15) to work well also for long maturities. By contrast, in the Hull and White (1987) model, where the variance $V_{t}$ follows a log-normal process without drift, $M_{2 c}$ and $M_{3 c}$ tend to infinity when $T$ increases and the series (11) fails to give the right price; cf. Hull and White (1987) and Gesser and Poncet (1997). The effect of moving to a mean reverting process from a log-normal process is to avoid that the variance explodes or goes to zero when $T$ increases.

Lewis (2000) derived a closed-form approximation for European option prices for a large class of stochastic volatility models including the GARCH diffusion model (7)-(8). Lewis's approximation formula for European option prices is based on second order Taylor expansion of some complex integrals around $c_{3}=0$; see Lewis (2000), p. 77-84. When $c_{3}=0, \bar{V}_{T}$ is deterministic and equals to $M_{1}^{g d}$. Indeed, it can be shown that Lewis's approximation is a particular case of our approximation (15) and is obtained by (15) neglecting terms $o\left(c_{3}^{2}\right)$. Therefore, for the GARCH diffusion model, our approximation is more accurate than the Lewis's one.

In the following section, by Monte Carlo simulations we study the accuracy of the approximation formula (15).

\section{Monte Carlo Simulations}

In order to verify the accuracy of the approximation (15) we compute by Monte Carlo simulations European option prices. The advantage of using Monte Carlo estimates is that the standard error of estimates is known. Precisely, we compute put option prices ${ }^{8}$ implied by (9) using the conditional Monte Carlo method; cf. Boyle, Broadie and Glasserman (1997).

Specifically, we divide the time interval $[0, T]$ into $s$ equal subintervals and we draw $s$ independent standard normal variables $\left(v_{i}\right)_{i=1, \ldots, s}$. We simulate the random variable $V_{t}$ in (8) over the discrete time $i T / s$, for $i=1, \ldots, s$, using the Milstein scheme (cf. Kloeden and Platen (1999))

$$
V_{i}=c_{1} \Delta t+V_{i-1}\left(1-c_{2}^{*} \Delta t+c_{3} \sqrt{\Delta t} v_{i}\right)+\frac{1}{2} c_{3}^{2} V_{i-1}^{2}\left(\left(\sqrt{\Delta t} v_{i}\right)^{2}-\Delta t\right)
$$

where $\Delta t:=T / s$. Then, we compute the Black and Scholes put option price $P_{b s}^{(n)}$ with squared volatility $s^{-1} \sum_{i=1}^{s} V_{i}$. Finally, iterating this procedure $N$ times we obtain the Monte Carlo

\footnotetext{
${ }^{8}$ Monte Carlo standard errors are generally smaller for put option prices than for call option prices as in the first case payoffs are bounded. Using the put-call parity call option prices are readily computed.
} 
estimate for the put option price

$$
P_{m c}:=N^{-1} \sum_{n=1}^{N} P_{b s}^{(n)},
$$

with the corresponding Monte Carlo standard error

$$
e_{m c}:=\frac{\sqrt{N^{-1} \sum_{n=1}^{N}\left(P_{b s}^{(n)}-P_{m c}\right)^{2}}}{\sqrt{N}} .
$$

When $N$ goes to infinity, $P_{m c}$ converges in probability to the put option price implied by (9). Notice that we do not need to simulate the price process $S$.

To simulate the variance process (8) we use parameter values inferred from empirical estimates of model (7)-(8). Typically, for currency and index daily log-returns the unconditional mean of $V, c_{1} / c_{2}^{*}$, ranges from 0.01 to 0.1 per year. The 'half life ${ }^{9}$ varies from few days to about a half year; cf. Chesney and Scott (1989), Taylor and Xu (1994), Xu and Taylor (1994), Guo $(1996,1998)$ and Fouque, Papanicolaou and Sircar (2000). This implies that $c_{2}^{*}$ ranges from 1 to 40. Moreover, empirical estimates of discrete GARCH(1,1)-M model on currency and index daily log-returns imply values of $c_{3}$ ranging from about 1 to 4 ; cf., for instance, Hull and White $(1987 \mathrm{a}, 1988)$ and Guo $(1996,1998)$. For stock log-returns, estimates of $c_{3}$ are generally smaller.

For the Monte Carlo simulations, we consider time to maturities for European put options ranging from 30 to 504 days. We wrote a Matlab code to run $N=10^{6}$ simulations. The computation time goes from about 14 hours for $T=30$ days to 15 hours for $T=504$ days on a PC Pentium IV 1GHz, running Windows XP.

In Table 1 we simulate the risk-adjusted variance process (8) using parameter values that we infer (cf. Nelson (1990)) from the GARCH(1,1) estimates in Guo (1996) for the dollar/yen exchange rates ${ }^{10}$, i.e. $c_{1}=0.16, c_{2}^{*}=18$ and $c_{3}=1.8$. The variance process is quickly mean reverting (the half life is about 10 days) and rather volatile, the two-standard deviation range for $V$ is from 0.003 to 0.016 ; see equations (6). Table 1 shows the Monte Carlo put price $P_{m c}$, the put price $\widetilde{P}^{g d}$ given by the series approximation (15), the pricing error $e_{p} \%$ defined as $e_{p} \%:=100 \times\left(P_{m c}-\widetilde{P}^{g d}\right) / P_{m c}$ and the Monte Carlo standard error $e_{m c}$. All errors are practically negligible across all strikes and maturities and the average error is $-0.025 \%$. Although the variance process is rather volatile, the high mean reversion rate $c_{2}^{*}$ implies that the integrated variance process $\bar{V}_{T}$ tends to stay around $\mathbb{E}\left[\bar{V}_{T} \mid V_{0}\right]$ and then the approximation (15) works well.

\footnotetext{
${ }^{9}$ The 'half life' is the time necessary after a shock to halve the deviation of $V_{t}$ from its run mean value, given that there are no more shocks. For this model the half life is equal to $\ln (2) / c_{2}^{*}$ years.

${ }^{10} \mathrm{As}$ in Guo (1996) we assume the volatility risk premium $\lambda(S, V, t)=0$.
} 
In Table 2 we simulate the variance process (8) using the risk-neutral parameters estimated by Melenberg and Werker (2001) for the Dutch EOE index. The volatility risk premium was estimated using European call options on the Dutch index. The correlation between price and volatility was negligible. The risk-neutral coefficients are $c_{1}=0.53, c_{2}^{*}=29.23$ and $c_{3}=3.65$. The run mean value of the variance is 0.018 and the two-standard deviation range for $V$ is $0-0.03$. Table 2 is organized as Table 1 . Also in this case pricing errors $e_{p} \%$ are almost always lower than $1 \%$ (except for one case). The average error is $-0.011 \%$.

In Table 3 and Table 4 we use parameter values that give reasonable variance process as discussed in Hull and White (1988). In Table 3 we set $c_{1}=0.18, c_{2}^{*}=2$ and $c_{3}=0.8$. The parameter value $c_{2}^{*}$ is quite small and implies a 'slow' mean reverting variance process (8), the half life of about 88 days. The unconditional mean and standard deviation of $V$ are 0.09 and 0.03 , respectively, and the two-standard deviation range for $V$ is $0.01-0.16$. As the volatility of $V_{t}$ is not too large, the process $\bar{V}_{T}$ tends to stay around $\mathbb{E}\left[\bar{V}_{T} \mid V_{0}\right]$ and hence the series approximation (15) is very accurate. The average pricing error is $-0.044 \%$. In Table 4 we set $c_{1}$ and $c_{2}^{*}$ as in Table 3 and $c_{3}=1.2$. This implies that the standard deviation of $\mathrm{V}$ is 0.06 and the two-standard deviation range for $V$ is $0-0.22$. Table 4 shows that pricing errors $e_{p} \%$ are still very small (the average pricing error is $-0.2 \%$ ) but slightly larger than in Table 3 as the variance process is more volatile than in the previous case.

Finally, in Table 5 we set $c_{1}=0.09, c_{2}^{*}=4$ and $c_{3}=1.2$ as in Lewis (2000). The unconditional mean of $V$ is 0.02 , the 'half life' is about 43 days and the two-standard deviation range for $V$ is 0.001-0.04. Also in this case errors $e_{p} \%$ are generally quite small and the average pricing error is $-0.018 \%$.

We simulate the variance process (8) also for other reasonable parameter choices (not reported here) and we found similar results. The approximation formula (15) induces pricing errors less than $1 \%$ for at the money options and less than $2 \%$ for out of the money options. Bid-ask spreads on currency option prices are larger than $2 \%$ of the prices for out of the money options and about $1 \%$ for more liquid, at the money currency options. Then, the approximation formula (15) gives accurate prices within the tolerance imposed by market frictions.

\section{$5 \quad$ Implied Volatility Surfaces}

In this section we study the implied volatility induced by the GARCH diffusion model (7)-(8), i.e. the volatility $\sigma_{i m p}^{2}$ which gives the Black and Scholes option price equals to the GARCH diffusion

option price, $C_{b s}\left(\sigma_{i m p}^{2}\right)=\widetilde{C}^{g d}$. Typically, to solve the implicit equation $C_{b s}\left(\sigma_{i m p}^{2}\right)=\widetilde{C}^{g d}$ the 
Newton-Raphson method is used ${ }^{11}$. Hence, we propose to compute $\sigma_{i m p}^{2}$ as

$$
\sigma_{i m p}^{2}=M_{1}^{g d}+\frac{\widetilde{C}^{g d}-C_{b s}\left(M_{1}^{g d}\right)}{\partial C_{b s} /\left.\partial \bar{V}_{T}\right|_{\bar{V}_{T}=M_{1}^{g d}}},
$$

that is a one-step Newton-Raphson algorithm starting at $M_{1}^{g d}$. As $\sigma_{i m p}^{2} \rightarrow M_{1}^{g d}$ when $T \rightarrow \infty$, $M_{1}^{g d}$ is a sensible starting point for the algorithm and one iteration gives very accurate results ${ }^{12}$. Given $\widetilde{C}^{g d}$ implementing (16) is straightforward and the model (7)-(8) can be easily calibrated to the market implied volatilities.

Renault and Touzi (1996) show that, for any stochastic volatility process, the assumption of no correlation between price and variance induces symmetric 'volatility smiles', i.e. symmetric shape with respect to the forward price of the implied volatility plotted as a function of the strike price; cf. also Hull and White (1987). The functional dependence of implied volatilities on time to maturities, i.e. the 'term structure patterns', depends on the specific variance process. In the following we qualitatively study the volatility smile and the term structure pattern induced by the GARCH diffusion model. As in Table 5 we set $c_{1}=0.09, c_{2}^{*}=4$ and $c_{3}=1.2$ and we compute the GARCH diffusion option prices (15). Then, by formula (16) we obtain the implied volatilities for different strikes and maturities. Figure 1 shows volatility smiles for time to maturities equal to 30, 60, 90 and 120 days. Figure 2 shows the volatility surface for time to maturity between 0 and 120 days and strike prices between 90 and 110. As expected, volatility smiles are quite symmetric with respect to the forward price. Moreover, the convexity of the volatility surface increases when the time to maturity decreases. These features of implied volatility surface were observed for all parameter choices (positive parameters). When the time to maturity increases the volatility surface flattens because the random variable $\bar{V}_{T}$ converges to the the run mean value $c_{1}^{*} / c_{2}^{*}$ by the Ergodic theorem and $\sigma_{i m p}^{2} \rightarrow c_{1}^{*} / c_{2}^{*}$ for all strike prices. These results are in qualitative agreement with the empirical evidence on volatility surfaces observed in currency option markets, where volatility smiles are quite symmetric with respect to the forward price, very pronounced at short maturities and almost flat for long maturities; cf., for instance, Chesney and Scott (1989), Melino and Turbull (1990), Taylor and Xu (1994), and Bollerslev and Zhou (2002).

\footnotetext{
${ }^{11}$ See for instance the Matlab function blsimpvdiv and the Mathematica function BlackScholesCallImpVol.

${ }^{12}$ We compared implied volatilities given by (16) with implied volatilities returned by the Matlab function blsimpvdiv and the errors were less than $0.01 \%$ for all the parameters used in Section 4.
} 


\section{Simple Estimators based on Nelson's Theory}

Inference on continuous time parameters of stochastic volatility models is an important issue in financial econometrics. The intractable likelihood functions and the unobservable volatility process prevent simple and efficient estimation procedures ${ }^{13}$. Nelson (1990) derived some moment conditions under which the discrete time GARCH(1,1)-M model (cf. Engle and Bollerslev 1986)) converges in distribution to the GARCH diffusion model (1)-(2). Such a convergence has been advocated by many authors (see, for instance, Engle and Lee (1996) and Lewis (2000)) to infer the continuous time parameters by the parameter estimates of the GARCH(1,1)-M model. As such models can be easily estimated, the previous inference procedures is very appealing. However, to our knowledge, the accuracy of the continuous time parameter estimates obtained by the discrete time parameter estimates have not been adequately verified.

In this section we investigate by Monte Carlo simulation how Nelson's theory works in practice and the convergence, under previous conditions, of the stochastic difference equations (17) to the stochastic differential equations (1)-(2) when $h$, i.e. the length of the discrete time interval between two observations, goes to zero.

The discrete time GARCH(1,1)-M model is

$$
\begin{aligned}
Y_{k h} & =Y_{(k-1) h}+\left[\mu-0.5 \sigma_{k h}^{2}\right] h+\sigma_{k h} Z_{k h} \\
\sigma_{(k+1) h}^{2} & =w^{(h)}+\beta^{(h)} \sigma_{k h}^{2}+\alpha^{(h)} \sigma_{k h}^{2} Z_{k h}^{2} h^{-1},
\end{aligned}
$$

where $Y_{k h}:=\log \left(S_{k h}\right), k \in \mathbb{N}, Z_{k h} \sim i . i . d . \mathcal{N}(0, h)$ and $\sigma_{k h}^{2}$ is the conditional variance of $Y_{k h}-Y_{(k-1) h}$. Using a moment matching procedure Nelson showed that, when $h \downarrow 0$, the sequence of continuous time version of (17) converges in distribution to the continuous time process (1)-(2). Nelson's procedure also yields the following relation between continuos and discrete time parameters up to $o(h)$,

$$
c_{1}=w^{(h)} / h, \quad c_{2}=1-\beta^{(h)}-\alpha^{(h)}, \quad c_{3}=\alpha^{(h)} \sqrt{2 / h},
$$

cf. Nelson (1990), pp. 15-18. Then, simple Maximum Likelihood estimates of model (17) allow to infer $c_{1}, c_{2}$ and $c_{3}$. Moreover, as $h$ shrinks the inference results should be more accurate. Using an Euler scheme ${ }^{14}$, we simulate 1,000 sample path realizations of the GARCH diffusion

\footnotetext{
${ }^{13}$ Several estimation methods have been proposed, such as the simulation based method of moments (Duffie and Singleton (1993)) or Bayesian Markov chain Monte Carlo methods (Jones (2003)). Surveys on stochastic volatility models including the estimation problem are given by, for instance, Bollerslev et al. (1994) and Ghysels et al. (1996).

${ }^{14}$ The Euler scheme for equations (1)-(2) used here is $\log \left(S_{i}\right)=\log \left(S_{i-1}\right)+\left[\mu-0.5 V_{i-1}\right] \Delta t+\sqrt{V_{i-1} \Delta t} \epsilon_{i}$ and $V_{i}=c_{1} \Delta t+V_{i-1}\left[1-c_{2} \Delta t+c_{3} \sqrt{\Delta t} v_{i}\right]$, where $\epsilon_{i}$ and $v_{i}$ denote independent standard normal random
} 
process $\left(S_{t}, V_{t}\right)_{t \in\left[0, T_{s m p}\right]}$, for the parameter choice $c_{1}=0.18, c_{2}=2, c_{3}=1.2$ (as in Table 4 ) and $T_{s m p}=20,40$ years. For each sample path, we estimate the model (17) using two-daily ( $h=2 / 360)$, daily $(h=1 / 360)$ and two-hourly $(h=1 / 3,000)$ log-returns and then we infer the continuous time parameters by formulae (18). The estimation results are given in Table 6 and Figure 3 and 4. As predicted by Nelson's theory, higher the sampling frequency more accurate the inference results are in terms of biases and root mean square errors. Obviously, results based on $T_{s m p}=40$ years are more accurate, but such a sample size is unrealistic for empirical studies. This simple and parsimonious estimation procedure provides still accurate inference results using $T_{s m p}=20$ years of simulated data. Other continuous time parameters lead to similar results. Hence, such a procedure may be used for inference on GARCH diffusion model parameters. More involved estimation procedures should be required to outperform the above results. Finally, when $T_{s m p}=40$ years, for two-daily estimates the sample size is large enough to overcome finite sample problems and regard the estimates as asymptotic ${ }^{15}$.

\section{Conclusions}

We derive analytically the first four conditional moments of the integrated variance under the GARCH diffusion model. Using these conditional moments we obtain an analytical closedform approximation formula $\widetilde{C}^{g d}(15)$ which allows us to price European options under the GARCH diffusion model. This formula can be easily implemented in any software package and provides option prices without any computational efforts. Monte Carlo simulations show that this approximation is accurate across different strikes and maturities for a large set of reasonable parameters. Finally, using the approximation formula (15) we study implied volatility surfaces induced by GARCH diffusion models. We find that volatility smiles and term structure patterns of implied volatilities are in qualitative agreement with volatility surfaces typically observed in the foreign exchange option markets.

Finally, we briefly investigate the inference on GARCH diffusion model parameters using an estimator based on Nelson's theory. We conclude that, considering the simple and parsimonious estimation procedure, inference results are rather accurate. Hence, this procedure may be used to infer GARCH diffusion model parameters.

variables and $\Delta t=1 /(360 \times 24)$. The sample path approximation to the diffusion can be made arbitrarily close by increasing the number of equally spaced increments per unit time interval. In this setting, the Milstein scheme is not advisable as multiple stochastic integrals can not be easily expressed in terms of increments of Brownian Motions; see for instance Kloeden and Platen (1999).

${ }^{15}$ We repeat the Monte Carlo study using $T_{s m p}=60$ years and we get similar results. 


\section{A Proof of Proposition 3.1}

In the following, we derive the first two conditional moments of the integrated variance $\bar{V}_{T}$ for the GARCH diffusion process,

$$
\bar{V}_{T}=\frac{V_{0}}{T} \int_{0}^{T} d t e^{-\left(c_{2}+\frac{1}{2} c_{3}^{2}\right) t} e^{c_{3} W_{t}}+\frac{c_{1}}{T} \int_{0}^{T} d t \int_{0}^{t} d s e^{\left(c_{2}+\frac{1}{2} c_{3}^{2}\right)(s-t)} e^{c_{3}\left(W_{t}-W_{s}\right)} .
$$

To prove Proposition 3.1 we recall that, if $w$ is a normal random variable $w \sim \mathcal{N}(0, t)$

$$
\mathbb{E}\left[e^{\lambda w}\right]=e^{\frac{\lambda^{2} t}{2}} .
$$

We also need the following lemma

\section{Lemma A.1}

$\forall x>y>0$,

$$
F(x, y)=e^{-\left(c_{2}+\frac{1}{2} c_{3}^{2}\right)(x+y)} \mathbb{E}\left[e^{c_{3}\left(W_{x}+W_{y}\right)}\right]=e^{-c_{2} x} e^{\left(c_{3}^{2}-c_{2}\right) y} .
$$

$\forall x>y>\alpha>0$,

$$
G(x, y, \alpha)=e^{-\left(c_{2}+\frac{1}{2} c_{3}^{2}\right)(x+y-\alpha)} \mathbb{E}\left[e^{c_{3}\left(W_{x}+W_{y}-W_{\alpha}\right)}\right]=e^{-c_{2} x} e^{\left(c_{3}^{2}-c_{2}\right) y} e^{\left(c_{2}-c_{3}^{2}\right) \alpha} .
$$

$\forall x>\alpha>y>0$,

$$
H(x, y, \alpha)=e^{-\left(c_{2}+\frac{1}{2} c_{3}^{2}\right)(x+y-\alpha)} \mathbb{E}\left[e^{c_{3}\left(W_{x}+W_{y}-W_{\alpha}\right)}\right]=e^{-c_{2}(x+y-\alpha)} .
$$

$\forall x>y>\alpha>\beta>0$,

$$
L(x, y, \alpha, \beta)=e^{-\left(c_{2}+\frac{1}{2} c_{3}^{2}\right)(x+y-\alpha-\beta)} \mathbb{E}\left[e^{c_{3}\left(W_{x}+W_{y}-W_{\alpha}-W_{\beta}\right)}\right]=e^{-c_{2} x} e^{\left(c_{3}^{2}-c_{2}\right) y} e^{\left(c_{2}-c_{3}^{2}\right) \alpha} e^{c_{2} \beta} .
$$

$\forall x>\alpha>y>\beta>0$,

$$
M(x, y, \alpha, \beta)=e^{-\left(c_{2}+\frac{1}{2} c_{3}^{2}\right)(x+y-\alpha-\beta)} \mathbb{E}\left[e^{c_{3}\left(W_{x}+W_{y}-W_{\alpha}-W_{\beta}\right)}\right]=e^{-c_{2}(x+y-\alpha-\beta)} .
$$

Proof. To prove $(21)$ we write $W_{x}+W_{y}=\left(W_{x}-W_{y}\right)+2 W_{y}$. As $\left(W_{x}-W_{y}\right)$ and $W_{y}$ are non-overlapping increments of the Brownian motion $W,\left(W_{x}-W_{y}\right) \sim \mathcal{N}(0, x-y)$ and $2 W_{y} \sim \mathcal{N}(0,4 y)$ one has

$$
\mathbb{E}\left[e^{c_{3}\left(W_{x}+W_{y}\right)}\right]=\mathbb{E}\left[e^{c_{3}\left(W_{x}-W_{y}\right)+2 W_{y}}\right]=\mathbb{E}\left[e^{c_{3}\left(W_{x}-W_{y}\right)}\right] \mathbb{E}\left[e^{c_{3} 2 W_{y}}\right]
$$

then formula (21) follows directly from (20).

To prove (22), use $W_{x}+W_{y}-W_{\alpha}=\left(W_{x}-W_{y}\right)+2\left(W_{y}-W_{\alpha}\right)+W_{\alpha}$.

To prove (23), use $W_{x}+W_{y}-W_{\alpha}=\left(W_{x}-W_{\alpha}\right)+W_{y}$.

To prove (24), use $W_{x}+W_{y}-W_{\alpha}-W_{\beta}=\left(W_{x}-W_{y}\right)+2\left(W_{y}-W_{\alpha}\right)+W_{\alpha}-W_{\beta}$.

To prove (25), use $W_{x}+W_{y}-W_{\alpha}-W_{\beta}=\left(W_{x}-W_{\alpha}\right)+\left(W_{y}-W_{\beta}\right)$. 


\section{A.1 First conditional moment}

The first conditional moment of $\bar{V}_{T}$ is given by

$$
\begin{aligned}
M_{1}^{g d} & :=\mathbb{E}\left[\bar{V}_{T} \mid V_{0}\right] \\
& =\frac{V_{0}}{T} \int_{0}^{T} d t e^{-\left(c_{2}+\frac{1}{2} c_{3}^{2}\right) t} \mathbb{E}\left[e^{c_{3} W_{t}}\right]+\frac{c_{1}}{T} \int_{0}^{T} d t \int_{0}^{t} d s e^{\left(c_{2}+\frac{1}{2} c_{3}^{2}\right)(s-t)} \mathbb{E}\left[e^{c_{3}\left(W_{t}-W_{s}\right)}\right] .
\end{aligned}
$$

As $W_{t} \sim \mathcal{N}(0, t)$ and $W_{t}-W_{s} \sim \mathcal{N}(0, t-s)$, using (20) we get the first conditional moment (13) in Proposition 3.1,

$$
\begin{aligned}
M_{1}^{g d} & :=\frac{V_{0}}{T} \int_{0}^{T} d t e^{-\left(c_{2}+\frac{1}{2} c_{3}^{2}\right) t} e^{\frac{1}{2} c_{3}^{2} t}+\frac{c_{1}}{T} \int_{0}^{T} d t \int_{0}^{t} d s e^{\left(c_{2}+\frac{1}{2} c_{3}^{2}\right)(s-t)} e^{\frac{1}{2} c_{3}^{2}(t-s)} \\
& =V_{0} \int_{0}^{T} e^{-c_{2} t} d t+c_{1} \int_{0}^{T} d t \int_{0}^{t} d s e^{c_{2}(s-t)}=\frac{V_{0}}{c_{2}}\left(\frac{1-e^{-c_{2} T}}{T}\right)+\frac{c_{1}}{T} \int_{0}^{T} \frac{1-e^{-c_{2} t}}{c_{2}} d t \\
& =\frac{c_{1}}{c_{2}}+\left(V_{0}-\frac{c_{1}}{c_{2}}\right) \frac{1-e^{-c_{2} T}}{c_{2} T} .
\end{aligned}
$$

\section{A.2 Second conditional moment}

The second conditional moment of $\bar{V}_{T}$ is given by

$$
\begin{aligned}
& \mathbb{E}\left[\bar{V}_{T}^{2} \mid V_{0}\right]=\mathbb{E}\left[\frac{1}{T^{2}} \int_{0}^{T} d r_{2} \int_{0}^{T} d r_{1}\left(V_{r_{1}} V_{r_{2}}\right)\right]=\frac{1}{T^{2}} \int_{0}^{T} d r_{2} \int_{0}^{T} d r_{1} \mathbb{E}\left[V_{r_{1}} V_{r_{2}}\right] \\
& =\frac{2 !}{T^{2}} \int_{0}^{T} d r_{2} \int_{0}^{r_{2}} d r_{1} \mathbb{E}\left[V_{r_{1}} V_{r_{2}}\right] \\
& =\frac{2 !}{T^{2}} \int_{0}^{T} d r_{2} \int_{0}^{r_{2}} d r_{1}(\mathbb{E}[A]+\mathbb{E}[B]+\mathbb{E}[C]+\mathbb{E}[D]),
\end{aligned}
$$

where

$$
\begin{aligned}
& A:=V_{0}^{2} e^{-\left(c_{2}+\frac{1}{2} c_{3}^{2}\right)\left(r_{1}+r_{2}\right)+c_{3}\left(W_{r_{1}}+W_{r_{2}}\right)} \\
& B:=c_{1} V_{0} e^{-\left(c_{2}+\frac{1}{2} c_{3}^{2}\right) r_{1}+c_{3} W_{r_{1}}} \int_{0}^{r_{2}} d s_{2} e^{\left(c_{2}+\frac{1}{2} c_{3}^{2}\right)\left(s_{2}-r_{2}\right)+c_{3}\left(W_{r_{2}}-W_{s_{2}}\right)}, \\
& C:=c_{1} V_{0} e^{-\left(c_{2}+\frac{1}{2} c_{3}^{2}\right) r_{2}+c_{3} W_{r_{2}}} \int_{0}^{r_{1}} d s_{1} e^{\left(c_{2}+\frac{1}{2} c_{3}^{2}\right)\left(s_{1}-r_{1}\right)+c_{3}\left(W_{r_{1}}-W_{s_{1}}\right)}, \\
& D:=c_{1}^{2} \int_{0}^{r_{1}} d s_{2} \int_{0}^{r_{2}} d s_{1} e^{\left(c_{2}+\frac{1}{2} c_{3}^{2}\right)\left(s_{1}-r_{1}+s_{2}-r_{2}\right)} e^{c_{3}\left(W_{r_{1}}-W_{s_{1}}+W_{r_{2}}-W_{s_{2}}\right)} .
\end{aligned}
$$

We compute each addend in (26).

- Calculation of

$$
\begin{aligned}
& \frac{2}{T^{2}} \int_{0}^{T} d r_{2} \int_{0}^{r_{2}} d r_{1} \mathbb{E}[A]= \\
& \quad \frac{2}{T^{2}} \int_{0}^{T} d r_{2} \int_{0}^{r_{2}} d r_{1} V_{0}^{2} e^{-\left(c_{2}+\frac{1}{2} c_{3}^{2}\right)\left(r_{1}+r_{2}\right)} \mathbb{E}\left[e^{c_{3}\left(W_{r_{1}}+W_{r_{2}}\right)}\right] .
\end{aligned}
$$


As $r_{2}>r_{1}>0$, we use formula (21) with $x=r_{2}$ and $y=r_{1}$

$$
\frac{2}{T^{2}} \int_{0}^{T} d r_{2} \int_{0}^{r_{2}} d r_{1} \mathbb{E}[A]=\frac{2 V_{0}^{2}}{T^{2}} \int_{0}^{T} d r_{2} \int_{0}^{r_{2}} d r_{1} F\left(r_{2}, r_{1}\right)
$$

and iterating integrations

$$
\frac{2}{T^{2}} \int_{0}^{T} d r_{2} \int_{0}^{r_{2}} d r_{1} \mathbb{E}[A]=\frac{2 V_{0}^{2}}{T^{2}}\left[\frac{e^{-\left(2 c_{2}-c_{3}^{2}\right) T}}{\left(c_{3}^{2}-2 c_{2}\right)\left(c_{3}^{2}-c_{2}\right)}+\frac{e^{-c_{2} T}}{c_{2}\left(c_{3}^{2}-c_{2}\right)}-\frac{1}{c_{2}\left(c_{3}^{2}-2 c_{2}\right)}\right]
$$

- Calculation of

$$
\begin{aligned}
& \frac{2}{T^{2}} \int_{0}^{T} d r_{2} \int_{0}^{r_{2}} d r_{1} \mathbb{E}[B]= \\
& \quad \frac{2 c_{1} V_{0}}{T^{2}} \int_{0}^{T} d r_{2} \int_{0}^{r_{2}} d r_{1} \int_{0}^{r_{1}} d s_{1} e^{-\left(c_{2}+\frac{1}{2} c_{3}^{2}\right)\left(r_{2}+r_{1}-s_{1}\right)} \mathbb{E}\left[e^{c_{3}\left(W_{r_{2}}+W_{r_{1}}-W_{s_{1}}\right)}\right]
\end{aligned}
$$

As $r_{2}>r_{1}>s_{1}>0$, we use formula (22) with $x=r_{2}, y=r_{1}$ and $\alpha=s_{1}$ and we get

$$
\begin{aligned}
& \frac{2}{T^{2}} \int_{0}^{T} d r_{2} \int_{0}^{r_{2}} d r_{1} \mathbb{E}[B]=\frac{2 c_{1} V_{0}}{T^{2}} \int_{0}^{T} d r_{2} \int_{0}^{r_{2}} d r_{1} \int_{0}^{r_{1}} d s_{1} G\left(r_{2}, r_{1}, s_{1}\right) \\
& \quad=\frac{c_{1} V_{0}}{T^{2} c_{2}^{4}\left(c_{2}-c_{3}^{2}\right)^{2}\left(-2 c_{2}+c_{3}^{2}\right)} \times \\
& \quad\left[-c_{2} e^{-T c_{2}}\left(-2 c_{2}+c_{3}^{2}\right)\left(c_{2}^{2}\left(-2+T c_{2}\right)+2 c_{2} c_{3}^{2}-\left(2+T c_{2}\right) c_{3}^{4}\right)\right. \\
& \left.\quad+c_{2}\left(c_{2}-c_{3}^{2}\right)^{2}\left(-2 c_{2}\left(-1+T c_{2}\right)+\left(-2+T c_{2}\right) c_{3}^{2}\right)+2 c_{2}^{4} e^{T\left(c_{3}^{2}-2 c_{2}\right)}\right] .
\end{aligned}
$$

- Calculation of

$$
\frac{2}{T^{2}} \int_{0}^{T} d r_{2} \int_{0}^{r_{2}} d r_{1} \mathbb{E}[C]
$$

Simply notice that

$$
\int_{0}^{T} d r_{2} \int_{0}^{T} d r_{1} \mathbb{E}[B]=\int_{0}^{T} d r_{2} \int_{0}^{T} d r_{1} \mathbb{E}[C] .
$$

- Calculation of

$$
\begin{aligned}
& \frac{2}{T^{2}} \int_{0}^{T} d r_{2} \int_{0}^{r_{2}} d r_{1} \mathbb{E}[D]= \\
& \quad \frac{2 c_{1}^{2}}{T^{2}} \int_{0}^{T} d r_{2} \int_{0}^{r_{2}} d r_{1} \int_{0}^{r_{2}} d s_{2} \int_{0}^{r_{1}} d s_{1}\left(e^{-\left(c_{2}+\frac{1}{2} c_{3}^{2}\right)\left(r_{2}+r_{1}-s_{2}-s_{1}\right)} \mathbb{E}\left[e^{c_{3}\left(W_{r_{2}}+W_{r_{1}}-W_{s_{1}}-W_{s_{2}}\right)}\right]\right) .
\end{aligned}
$$


We divide the integration domain of $s_{2}$ and $s_{1}$ as follows

$$
\begin{aligned}
& \frac{2}{T^{2}} \int_{0}^{T} d r_{2} \int_{0}^{r_{2}} d r_{1} \mathbb{E}[D]= \\
& \quad \frac{2 c_{1}^{2}}{T^{2}} \int_{0}^{T} d r_{2} \int_{0}^{r_{2}} d r_{1} \int_{0}^{r_{1}} d s_{2} \int_{0}^{s_{2}} d s_{1}(\ldots)+ \\
& \quad+\frac{2 c_{1}^{2}}{T^{2}} \int_{0}^{T} d r_{2} \int_{0}^{r_{2}} d r_{1} \int_{0}^{r_{1}} d s_{2} \int_{s_{2}}^{r_{1}} d s_{1}(\ldots)+ \\
& \quad+\frac{2 c_{1}^{2}}{T^{2}} \int_{0}^{T} d r_{2} \int_{0}^{r_{2}} d r_{1} \int_{r_{1}}^{r_{2}} d s_{2} \int_{0}^{r_{1}} d s_{1}(\ldots) .
\end{aligned}
$$

The previous partition allows us to use

formula (24) with $x=r_{2}, y=r_{1}, \alpha=s_{2}, \beta=s_{1}$ in (30) as $T>r_{2}>r_{1}>s_{2}>s_{1}>0$;

formula (24) with $x=r_{2}, y=r_{1}, \alpha=s_{1}, \beta=s_{2}$ in (31) as $T>r_{2}>r_{1}>s_{1}>s_{2}>0$;

formula (25) with $x=r_{2}, y=r_{1}, \alpha=s_{2}, \beta=s_{1}$ in (32) as $T>r_{2}>s_{2}>r_{1}>s_{1}>0$; then

$$
\begin{aligned}
& \frac{2}{T^{2}} \int_{0}^{T} d r_{2} \int_{0}^{r_{2}} d r_{1} \mathbb{E}[D]= \\
& \quad=\frac{2 c_{1}^{2}}{T^{2}} \int_{0}^{T} d r_{2} \int_{0}^{r_{2}} d r_{1} \int_{0}^{r_{1}} d s_{2} \int_{0}^{s_{2}} d s_{1} L\left(r_{2}, r_{1}, s_{2}, s_{1}\right) \\
& \quad+\frac{2 c_{1}^{2}}{T^{2}} \int_{0}^{T} d r_{2} \int_{0}^{r_{2}} d r_{1} \int_{0}^{r_{1}} d s_{2} \int_{s_{2}}^{r_{1}} d s_{1} L\left(r_{2}, r_{1}, s_{1}, s_{2}\right) \\
& \quad+\frac{2 c_{1}^{2}}{T^{2}} \int_{0}^{T} d r_{2} \int_{0}^{r_{2}} d r_{1} \int_{r_{1}}^{r_{2}} d s_{2} \int_{0}^{r_{1}} d s_{1} M\left(r_{2}, r_{1}, s_{2}, s_{1}\right)
\end{aligned}
$$

and iterating integrations

$$
\begin{aligned}
& \frac{2}{T^{2}} \int_{0}^{T} \int_{0}^{r_{2}} \mathbb{E}[D] d r_{1} d r_{2}=\frac{c_{1}^{2}}{T^{2} c_{2}^{4}\left(c_{2}-c_{3}^{2}\right)^{2}\left(-2 c_{2}+c_{3}^{2}\right)^{2}} \times \\
& \quad\left[-2 e^{-T c_{2}}\left(-2 c_{2}+c_{3}^{2}\right)^{2}\left(c_{2}^{2}-T c_{2}^{3}-2 c_{2} c_{3}^{2}+\left(3+T c_{2}\right) c_{3}^{4}\right)\right. \\
& \quad+\left(c_{2}-c_{3}^{2}\right)^{2}\left(\left(4 c_{2}^{2}\left(-1+T c_{2}\right)^{2}-4 c_{2}\left(4+T c_{2}\left(-3+T c_{2}\right)\right) c_{3}^{2}+\left(6+T c_{2}\left(-4+T c_{2}\right)\right) c_{3}^{4}\right)+\right. \\
& \left.\quad+4 e^{T\left(c_{3}^{2}-2 c_{2}\right)} c_{2}^{4}\right] .
\end{aligned}
$$


Summing (27), (28), (29) and (33) we get the second conditional moment of $\bar{V}_{T}$ :

$$
\begin{aligned}
M_{2}^{g d}:=\mathbb{E}\left[\bar{V}_{T}^{2} \mid V_{0}\right]= & \frac{1}{T^{2} c_{2}^{4}\left(c_{2}-c_{3}^{2}\right)^{2}\left(-2 c_{2}+c_{3}^{2}\right)^{2}} \\
& {\left[e ^ { - 2 T c _ { 2 } } \left(-2 e^{T c_{2}}\left(-2 c_{2}+c_{3}^{2}\right)^{2}\right.\right.} \\
& \left(c_{1}^{2}\left(c_{2}^{2}-T c_{2}^{3}-2 c_{2} c_{3}^{2}+\left(3+T c_{2}\right) c_{3}^{4}\right)\right. \\
& +c_{1} c_{2}\left(c_{2}^{2}\left(-2+T c_{2}\right)+2 c_{2} c_{3}^{2}-\left(2+T c_{2}\right) c_{3}^{4}\right) V_{0}+ \\
& \left.c_{2}^{3}\left(c_{2}-c_{3}^{2}\right) V_{0}^{2}\right)+e^{2 T c_{2}}\left(c_{2}-c_{3}^{2}\right)^{2}\left(c _ { 1 } ^ { 2 } \left(4 c_{2}^{2}\left(-1+T c_{2}\right)^{2}\right.\right. \\
& \left.-4 c_{2}\left(4+T c_{2}\left(-3+T c_{2}\right)\right) c_{3}^{2}+\left(6+T c_{2}\left(-4+T c_{2}\right)\right) c_{3}^{4}\right)+ \\
& 2 c_{1} c_{2}\left(2 c_{2}-c_{3}^{2}\right)\left(2 c_{2}\left(-1+T c_{2}\right)-\left(-2+T c_{2}\right) c_{3}^{2}\right) \\
& \left.V_{0}+2 c_{2}^{3}\left(2 c_{2}-c_{3}^{2}\right) V_{0}^{2}\right)+2 e^{T c_{3}^{2}} c_{2}^{4}\left(2 c_{1}^{2}-2 c_{1}\left(2 c_{2}-c_{3}^{2}\right) V_{0}+\right. \\
& \left.\left.\left.\left(2 c_{2}^{2}-3 c_{2} c_{3}^{2}+c_{3}^{4}\right) V_{0}^{2}\right)\right)\right] .
\end{aligned}
$$

The second central conditional moment of $\bar{V}_{T}, M_{2 c}^{g d}$, stated in (14) Proposition 3.1 is given by $M_{2 c}^{g d}=M_{2}^{g d}-\left(M_{1}^{g d}\right)^{2}$. 


\begin{tabular}{|c|c|c|c|c|c|}
\hline maturity & strike & $P_{m c}$ & $\widetilde{P}^{g d}$ & $e_{p} \%$ & $e_{m c} \times 10^{4}$ \\
\hline \multirow{5}{*}{30 days } & 90 & 0.0008 & 0.0008 & -0.6840 & 0.0129 \\
\hline & 95 & 0.0800 & 0.0800 & 0.1025 & 0.3610 \\
\hline & 100 & 1.2921 & 1.2924 & -0.0267 & 1.1997 \\
\hline & 105 & 5.0999 & 5.0998 & 0.0020 & 0.4231 \\
\hline & 110 & 10.0023 & 10.0024 & -0.0002 & 0.0294 \\
\hline \multirow{5}{*}{60 days } & 90 & 0.0184 & 0.0184 & -0.2445 & 0.1330 \\
\hline & 95 & 0.2993 & 0.2992 & 0.0199 & 0.8289 \\
\hline & 100 & 1.8284 & 1.8290 & -0.0281 & 1.5411 \\
\hline & 105 & 5.3497 & 5.3496 & 0.0008 & 0.9215 \\
\hline & 110 & 10.0363 & 10.0364 & -0.0002 & 0.2224 \\
\hline \multirow{5}{*}{90 days } & 90 & 0.0664 & 0.0664 & 0.0189 & 0.30666 \\
\hline & 95 & 0.5401 & 0.5401 & -0.0003 & 1.0964 \\
\hline & 100 & 2.2411 & 2.2414 & -0.0166 & 1.678 \\
\hline & 105 & 5.6155 & 5.6155 & -0.0003 & 1.1971 \\
\hline & 110 & 10.1131 & 10.1131 & 0.0004 & 0.45367 \\
\hline \multirow{5}{*}{120 days } & 90 & 0.1393 & 0.1393 & 0.0210 & 0.47265 \\
\hline & 95 & 0.7760 & 0.7760 & -0.0093 & 1.2603 \\
\hline & 100 & 2.5890 & 2.5893 & -0.0125 & 1.7489 \\
\hline & 105 & 5.8721 & 5.8722 & -0.0015 & 1.3634 \\
\hline & 110 & 10.2187 & 10.2187 & 0.0004 & 0.65406 \\
\hline \multirow{5}{*}{180 days } & 90 & 0.3295 & 0.3294 & 0.0171 & 0.7371 \\
\hline & 95 & 1.2163 & 1.2163 & -0.0038 & 1.4470 \\
\hline & 100 & 3.1727 & 3.1729 & -0.0052 & 1.8186 \\
\hline & 105 & 6.3464 & 6.3465 & -0.0009 & 1.5509 \\
\hline & 110 & 10.4734 & 10.4734 & 0.0005 & 0.9500 \\
\hline \multirow{5}{*}{252 days } & 90 & 0.5928 & 0.5929 & -0.0096 & 0.9571 \\
\hline & 95 & 1.6919 & 1.6921 & -0.0108 & 1.5684 \\
\hline & 100 & 3.7550 & 3.7553 & -0.0071 & 1.8615 \\
\hline & 105 & 6.8550 & 6.8552 & -0.0029 & 1.1821 \\
\hline & 110 & 10.8062 & 10.8063 & -0.0007 & 1.1863 \\
\hline \multirow{5}{*}{504 days } & 90 & 1.5438 & 1.5440 & -0.0101 & 1.3327 \\
\hline & 95 & 3.0577 & 3.0579 & -0.0076 & 1.7336 \\
\hline & 100 & 5.3116 & 5.3119 & -0.0050 & 1.9141 \\
\hline & 105 & 8.3052 & 8.3054 & -0.0029 & 1.8354 \\
\hline & 110 & 11.9443 & 2011.9445 & -0.0015 & 1.5584 \\
\hline
\end{tabular}

Table 1: $P_{m c}$ Monte Carlo put prices computed by $N=10^{6}$ simulations; $\widetilde{P}^{g d}$ put prices given 


\begin{tabular}{|c|c|c|c|c|c|}
\hline maturity & strike & $P_{m c}$ & $\widetilde{P}^{g d}$ & $e_{p} \%$ & $e_{m c} \times 10^{4}$ \\
\hline \multirow{5}{*}{30 days } & 90 & 0.0236 & 0.0240 & -1.5083 & 0.3503 \\
\hline & 95 & 0.3093 & 0.3071 & 0.7134 & 1.5033 \\
\hline & 100 & 1.8344 & 1.8389 & -0.2473 & 2.6253 \\
\hline & 105 & 5.3602 & 5.3580 & 0.0398 & 1.6594 \\
\hline & 110 & 10.0436 & 10.0435 & 0.0016 & 0.5242 \\
\hline \multirow{5}{*}{60 days } & 90 & 0.1508 & 0.1495 & 0.8793 & 0.9954 \\
\hline & 95 & 0.7899 & 0.7898 & 0.0177 & 2.3314 \\
\hline & 100 & 2.6014 & 2.6052 & -0.1464 & 3.1471 \\
\hline & 105 & 5.8867 & 5.8868 & -0.0017 & 2.5150 \\
\hline & 110 & 10.2325 & 10.2307 & 0.0176 & 1.3279 \\
\hline \multirow{5}{*}{90 days } & 90 & 0.3449 & 0.3438 & 0.3171 & 1.4788 \\
\hline & 95 & 1.2348 & 1.2355 & -0.0563 & 2.7146 \\
\hline & 100 & 3.1915 & 3.1942 & -0.0859 & 3.3572 \\
\hline & 105 & 6.3659 & 6.3668 & -0.0139 & 2.9048 \\
\hline & 110 & 10.4914 & 10.4903 & 0.0108 & 1.8716 \\
\hline \multirow{5}{*}{120 days } & 90 & 0.5668 & 0.5661 & 0.1128 & 1.8267 \\
\hline & 95 & 1.6394 & 1.6403 & -0.0526 & 2.9359 \\
\hline & 100 & 3.6891 & 3.6911 & -0.0555 & 3.4717 \\
\hline & 105 & 6.7987 & 6.7997 & -0.0147 & 3.1284 \\
\hline & 110 & 10.7723 & 10.7717 & 0.0049 & 2.2435 \\
\hline \multirow{5}{*}{180 days } & 90 & 1.0310 & 1.0311 & -0.0083 & 2.2774 \\
\hline & 95 & 2.3550 & 2.3561 & -0.0457 & 3.1795 \\
\hline & 100 & 4.5229 & 4.5245 & -0.0361 & 3.5916 \\
\hline & 105 & 7.5601 & 7.5613 & -0.0156 & 3.3733 \\
\hline & 110 & 11.3372 & 11.3375 & -0.0024 & 2.7073 \\
\hline \multirow{5}{*}{252 days } & 90 & 1.5777 & 1.5780 & -0.0217 & 2.6024 \\
\hline & 95 & 3.0980 & 3.0990 & -0.0314 & 3.3406 \\
\hline & 100 & 5.3549 & 5.3561 & -0.0239 & 3.6742 \\
\hline & 105 & 8.3478 & 8.3488 & -0.0126 & 3.5354 \\
\hline & 110 & 11.9831 & 11.9836 & -0.0042 & 3.0333 \\
\hline \multirow{5}{*}{504 days } & 90 & 3.2715 & 3.2724 & -0.0271 & 3.0858 \\
\hline & 95 & 5.1528 & 5.1540 & -0.0228 & 3.5523 \\
\hline & 100 & 7.5754 & 7.5767 & -0.0172 & 3.7759 \\
\hline & 105 & 10.5187 & 10.5199 & -0.0118 & 3.7472 \\
\hline & 110 & 13.9310 & 113.9320 & -0.0075 & 3.5050 \\
\hline
\end{tabular}

Table 2: $P_{m c}$ Monte Carlo put prices computed by $N=10^{6}$ simulations; $\widetilde{P}^{g d}$ put prices given 


\begin{tabular}{|c|c|c|c|c|c|}
\hline maturity & strike & $P_{m c}$ & $\widetilde{P}^{g d}$ & $e_{p} \%$ & $e_{m c} \times 10^{4}$ \\
\hline \multirow{5}{*}{30 days } & 90 & 0.7901 & 0.7900 & 0.0099 & 1.6735 \\
\hline & 95 & 2.0011 & 2.0010 & 0.0062 & 2.5395 \\
\hline & 100 & 4.1169 & 4.1169 & 0.0022 & 2.9438 \\
\hline & 105 & 7.1841 & 7.1840 & 0.0018 & 2.7010 \\
\hline & 110 & 11.0473 & 11.0472 & 0.0010 & 2.0293 \\
\hline \multirow{5}{*}{60 days } & 90 & 1.9061 & 1.9061 & -0.0017 & 4.0187 \\
\hline & 95 & 3.5125 & 3.5133 & -0.0222 & 5.0139 \\
\hline & 100 & 5.8083 & 5.8095 & -0.0199 & 5.4663 \\
\hline & 105 & 8.7862 & 8.7871 & -0.0097 & 5.3017 \\
\hline & 110 & 12.3644 & 12.3646 & -0.0015 & 4.6523 \\
\hline \multirow{5}{*}{90 days } & 90 & 2.8985 & 2.8990 & -0.0155 & 6.0747 \\
\hline & 95 & 4.7109 & 4.7128 & -0.0390 & 7.0986 \\
\hline & 100 & 7.1015 & 7.1040 & -0.0346 & 7.5819 \\
\hline & 105 & 10.0522 & 10.0542 & -0.0198 & 7.4915 \\
\hline & 110 & 13.5044 & 13.5052 & -0.0059 & 6.9242 \\
\hline \multirow{5}{*}{120 days } & 90 & 3.7858 & 3.7873 & -0.0410 & 7.8285 \\
\hline & 95 & 5.7337 & 5.7372 & -0.0606 & 8.8537 \\
\hline & 100 & 8.1892 & 8.1935 & -0.0523 & 9.3602 \\
\hline & 105 & 11.1311 & 11.1348 & -0.0334 & 9.3346 \\
\hline & 110 & 14.5125 & 14.5147 & -0.0148 & 8.8543 \\
\hline \multirow{5}{*}{180 days } & 90 & 5.3378 & 5.3424 & -0.0868 & 10.6045 \\
\hline & 95 & 7.4645 & 7.4717 & -0.0963 & 11.6077 \\
\hline & 100 & 10.0110 & 10.0192 & -0.0824 & 12.1468 \\
\hline & 105 & 12.9552 & 12.9628 & -0.0590 & 12.2263 \\
\hline & 110 & 16.2621 & 16.2678 & -0.0351 & 11.9015 \\
\hline \multirow{5}{*}{252 days } & 90 & 6.9387 & 6.9458 & -0.1024 & 13.0138 \\
\hline & 95 & 9.2060 & 9.2158 & -0.1065 & 13.9836 \\
\hline & 100 & 11.8297 & 11.8406 & -0.0924 & 14.5487 \\
\hline & 105 & 14.7891 & 14.7995 & -0.0702 & 14.7208 \\
\hline & 110 & 18.0562 & 18.0646 & -0.0469 & 14.5415 \\
\hline \multirow{5}{*}{504 days } & 90 & 11.3404 & 11.3521 & -0.1031 & 17.3351 \\
\hline & 95 & 13.8865 & 13.9001 & -0.0982 & 18.2109 \\
\hline & 100 & 16.6835 & 16.6980 & -0.0871 & 18.8137 \\
\hline & 105 & 19.7148 & 19.7292 & -0.0730 & 19.1580 \\
\hline & 110 & 22.9623 & 222.9757 & -0.0581 & 19.2664 \\
\hline
\end{tabular}

Table 3: $P_{m c}$ Monte Carlo put prices computed by $N=10^{6}$ simulations; $\widetilde{P}^{g d}$ put prices given 


\begin{tabular}{|c|c|c|c|c|c|}
\hline maturity & strike & $P_{m c}$ & $\widetilde{P}^{g d}$ & $e_{p} \%$ & $e_{m c} \times 10^{4}$ \\
\hline \multirow{5}{*}{30 days } & 90 & 0.7901 & 0.7894 & 0.0882 & 2.5219 \\
\hline & 95 & 1.9925 & 1.9924 & 0.0036 & 3.8025 \\
\hline & 100 & 4.1038 & 4.1043 & -0.0133 & 4.4041 \\
\hline & 105 & 7.1747 & 7.1746 & 0.0004 & 4.0438 \\
\hline & 110 & 11.0457 & 11.0450 & 0.0067 & 3.0519 \\
\hline \multirow{5}{*}{60 days } & 90 & 1.8942 & 1.8934 & 0.0395 & 6.0288 \\
\hline & 95 & 3.4865 & 3.4906 & -0.1182 & 7.4909 \\
\hline & 100 & 5.7761 & 5.7826 & -0.1131 & 8.1602 \\
\hline & 105 & 8.7583 & 8.7629 & -0.0518 & 7.9202 \\
\hline & 110 & 12.3481 & 12.3483 & -0.0011 & 6.9710 \\
\hline \multirow{5}{*}{90 days } & 90 & 2.8713 & 2.8737 & -0.0855 & 9.0975 \\
\hline & 95 & 4.6673 & 4.6791 & -0.2543 & 10.5933 \\
\hline & 100 & 7.0509 & 7.0669 & -0.2268 & 11.3051 \\
\hline & 105 & 10.0057 & 10.0186 & -0.1283 & 11.1786 \\
\hline & 110 & 13.4706 & 13.4754 & -0.0353 & 10.3598 \\
\hline \multirow{5}{*}{120 days } & 90 & 3.7432 & 3.7529 & -0.2592 & 11.7084 \\
\hline & 95 & 5.6738 & 5.6972 & -0.4126 & 13.1994 \\
\hline & 100 & 8.1220 & 8.1511 & -0.3588 & 13.9428 \\
\hline & 105 & 11.0676 & 11.0927 & -0.2267 & 13.9152 \\
\hline & 110 & 14.4615 & 14.4753 & -0.0955 & 13.2316 \\
\hline \multirow{5}{*}{180 days } & 90 & 5.2682 & 5.3010 & -0.6238 & 15.8534 \\
\hline & 95 & 7.3776 & 7.4311 & -0.7247 & 17.3052 \\
\hline & 100 & 9.9165 & 9.9784 & -0.6242 & 18.0949 \\
\hline & 105 & 12.8633 & 12.9202 & -0.4422 & 18.2261 \\
\hline & 110 & 16.1813 & 16.2224 & -0.2536 & 17.7801 \\
\hline \multirow{5}{*}{252 days } & 90 & 6.8458 & 6.9104 & -0.9427 & 19.4864 \\
\hline & 95 & 9.0969 & 9.1869 & -0.9895 & 20.8891 \\
\hline & 100 & 11.7133 & 11.8138 & -0.8583 & 21.7182 \\
\hline & 105 & 14.6740 & 14.7694 & -0.6502 & 21.9888 \\
\hline & 110 & 17.9501 & 18.0272 & -0.4297 & 21.7612 \\
\hline \multirow{5}{*}{504 days } & 90 & 11.2078 & 11.3528 & -1.2940 & 26.2295 \\
\hline & 95 & 13.7415 & 13.9123 & -1.2428 & 27.5161 \\
\hline & 100 & 16.5319 & 16.7143 & -1.1035 & 28.4149 \\
\hline & 105 & 19.5621 & 19.7423 & -0.9214 & 28.9460 \\
\hline & 110 & 22.8135 & 322.9793 & -0.7267 & 29.1419 \\
\hline
\end{tabular}

Table 4: $P_{m c}$ Monte Carlo put prices computed by $N=10^{6}$ simulations; $\widetilde{P}^{g d}$ put prices given 


\begin{tabular}{|c|c|c|c|c|c|}
\hline maturity & strike & $P_{m c}$ & $\widetilde{P}^{g d}$ & $e_{p} \%$ & $e_{m c} \times 10^{4}$ \\
\hline \multirow{5}{*}{30 days } & 90 & 0.0416 & 0.0418 & -0.4024 & 0.2796 \\
\hline & 95 & 0.4271 & 0.4269 & 0.0559 & 1.2305 \\
\hline & 100 & 2.0543 & 2.0546 & -0.0118 & 2.0377 \\
\hline & 105 & 5.4912 & 5.4910 & 0.0044 & 1.3532 \\
\hline & 110 & 10.0743 & 10.0744 & -0.0011 & 0.4321 \\
\hline \multirow{5}{*}{60 days } & 90 & 0.2403 & 0.2395 & 0.3186 & 1.2698 \\
\hline & 95 & 1.0088 & 1.0088 & 0.0013 & 2.7060 \\
\hline & 100 & 2.8976 & 2.8995 & -0.0661 & 3.5178 \\
\hline & 105 & 6.1229 & 6.1230 & -0.0019 & 2.9091 \\
\hline & 110 & 10.3537 & 10.3527 & 0.0097 & 1.6629 \\
\hline \multirow{5}{*}{90 days } & 90 & 0.5059 & 0.5040 & 0.3736 & 2.2925 \\
\hline & 95 & 1.5236 & 1.5243 & -0.0482 & 3.8280 \\
\hline & 100 & 3.5450 & 3.5484 & -0.0953 & 4.5905 \\
\hline & 105 & 6.6746 & 6.6756 & -0.0146 & 4.0842 \\
\hline & 110 & 10.6943 & 10.6924 & 0.0180 & 2.8388 \\
\hline \multirow{5}{*}{120 days } & 90 & 0.7895 & 0.7873 & 0.2753 & 3.1594 \\
\hline & 95 & 1.9843 & 1.9862 & -0.0935 & 4.6713 \\
\hline & 100 & 4.0917 & 4.0965 & -0.1164 & 5.3827 \\
\hline & 105 & 7.1657 & 7.1679 & -0.0305 & 4.9653 \\
\hline & 110 & 11.0435 & 11.0416 & 0.0170 & 3.8040 \\
\hline \multirow{5}{*}{180 days } & 90 & 1.3561 & 1.3550 & 0.0806 & 4.4457 \\
\hline & 95 & 2.7925 & 2.7964 & -0.1395 & 5.8221 \\
\hline & 100 & 5.0114 & 5.0181 & -0.1319 & 6.4495 \\
\hline & 105 & 8.0238 & 8.0281 & -0.0539 & 6.1656 \\
\hline & 110 & 11.7203 & 11.7200 & 0.0026 & 5.2043 \\
\hline \multirow{5}{*}{252 days } & 90 & 2.0035 & 2.0036 & -0.0071 & 5.4574 \\
\hline & 95 & 3.6288 & 3.6333 & -0.1240 & 6.6719 \\
\hline & 100 & 5.9334 & 5.9400 & -0.1108 & 7.2297 \\
\hline & 105 & 8.9091 & 8.9140 & -0.0551 & 7.0508 \\
\hline & 110 & 12.4759 & 12.4770 & -0.0084 & 6.2867 \\
\hline \multirow{5}{*}{504 days } & 90 & 3.9613 & 3.9637 & -0.0605 & 7.0744 \\
\hline & 95 & 5.9352 & 5.9394 & -0.0710 & 7.9420 \\
\hline & 100 & 8.4032 & 8.4082 & -0.0592 & 8.3767 \\
\hline & 105 & 11.3436 & 11.3481 & -0.0396 & 8.3715 \\
\hline & 110 & 14.7118 & $\$ 414.7148$ & -0.0208 & 7.9875 \\
\hline
\end{tabular}

Table 5: $P_{m c}$ Monte Carlo put prices computed by $N=10^{6}$ simulations; $\widetilde{P}^{g d}$ put prices given 


\begin{tabular}{|c|c|c|c|c|c|c|}
\hline & \multicolumn{2}{|c|}{$c_{1}=0.18$} & \multicolumn{2}{c|}{$c_{2}=2$} & \multicolumn{2}{c|}{$c_{3}=1.2$} \\
\hline freq. & bias & rmse & bias & rmse & bias & rmse \\
\hline 2 days & 0.0442 & 0.0900 & 0.5814 & 1.2041 & -0.1379 & 0.2185 \\
\hline 1 day & 0.0351 & 0.0721 & 0.4720 & 1.0035 & -0.1003 & 0.1618 \\
\hline 2 hours & 0.0213 & 0.0505 & 0.3086 & 0.7758 & -0.0257 & 0.0606 \\
\hline \hline 2 days & 0.0283 & 0.0550 & 0.3531 & 0.7409 & -0.1296 & 0.1718 \\
\hline 1 day & 0.0232 & 0.0465 & 0.2939 & 0.6377 & -0.0928 & 0.1279 \\
\hline 2 hours & 0.0111 & 0.0302 & 0.1480 & 0.4692 & -0.0252 & 0.0470 \\
\hline
\end{tabular}

Table 6: Biases and root mean square errors (RMSE) of the continuous time parameters $c_{1}=$ $0.18, c_{2}=2$ and $c_{3}=1.2$ of the GARCH diffusion model (1)-(2) inferred by the parameter estimates of the discre time GARCH-M model (17) at two-daily, daily and two-hourly sampling frequencies. First panel: sample size 20 years; second panel: sample size 40 years. 

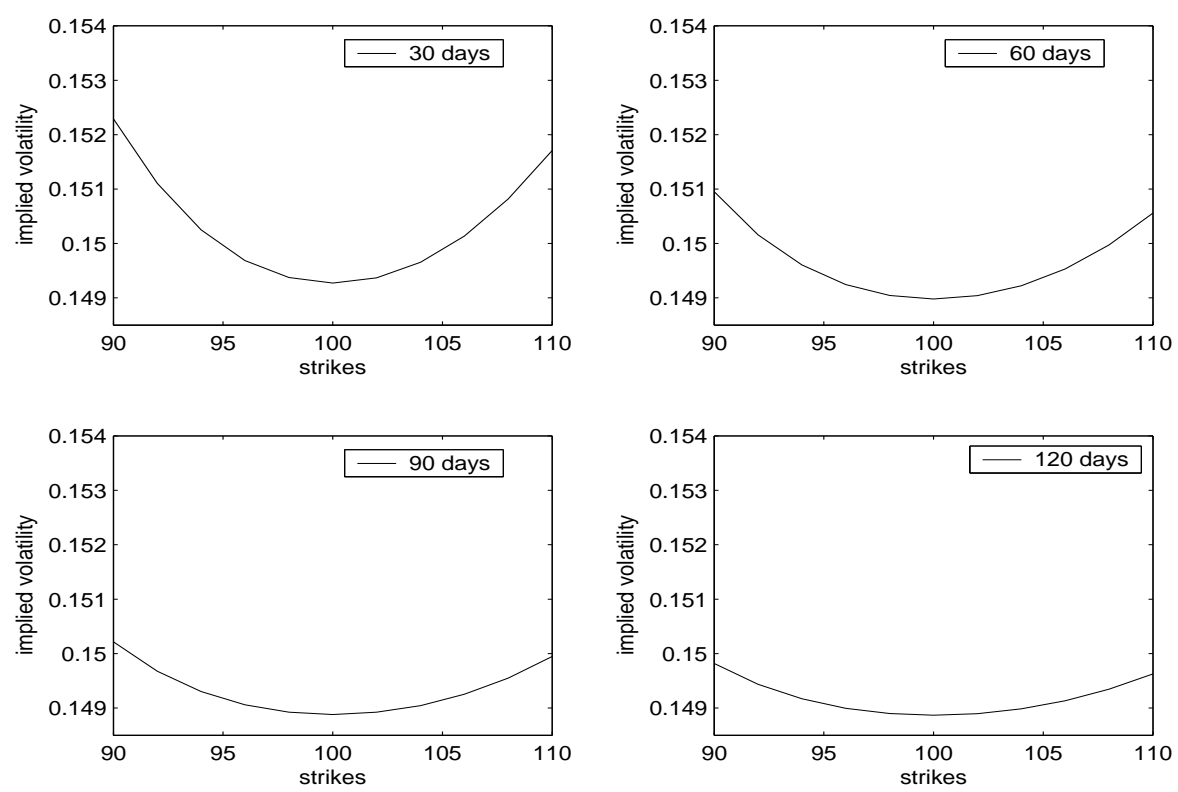

Figure 1: Volatility smiles for maturities of $30,60,90$ and 120 days and the parameter choice $S_{0}=100$, $r=0, d=0 ; d V=(0.09-4 V) d t+1.2 V d W, V_{0}=0.0225$, as in Table 5.

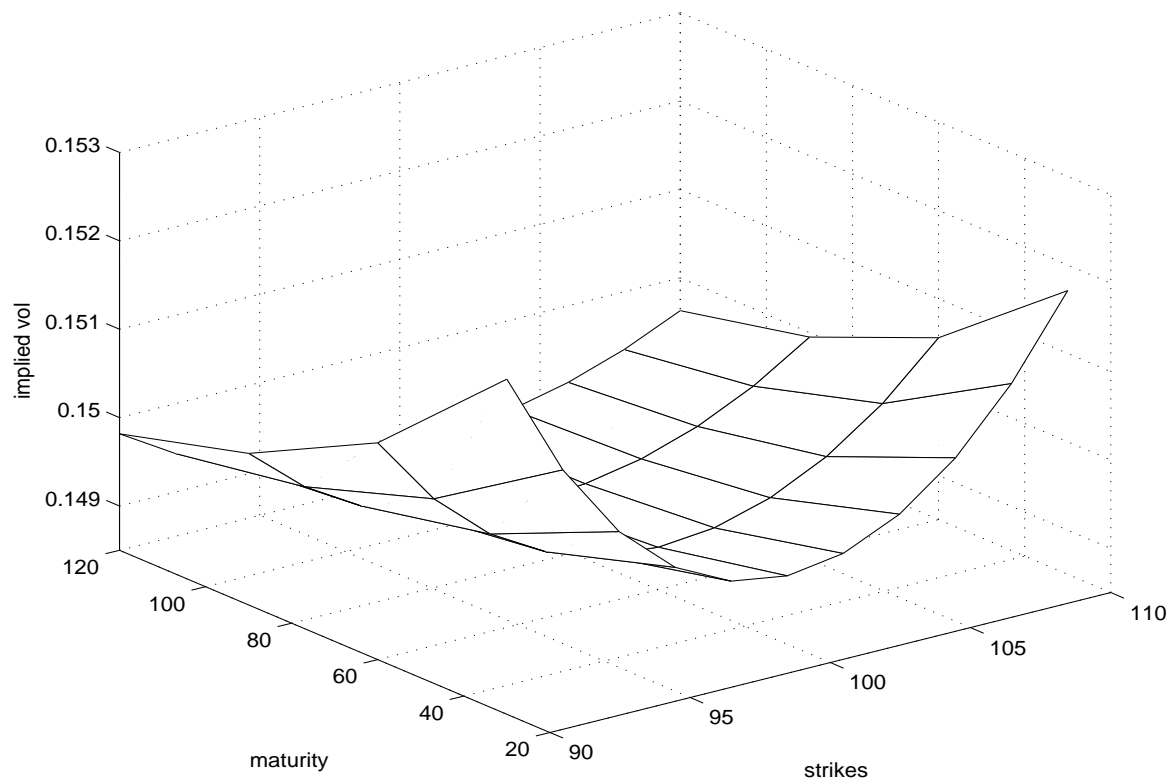

Figure 2: Volatility Surface for maturities $T \in[30,120]$ days, strikes $K \in[90,110]$ and the parameter choice $S_{0}=100, r=0, d=0 ; d V=(0.09-4 V) d t+1.2 V d W, V_{0}=0.0225$, as in Table 5. 

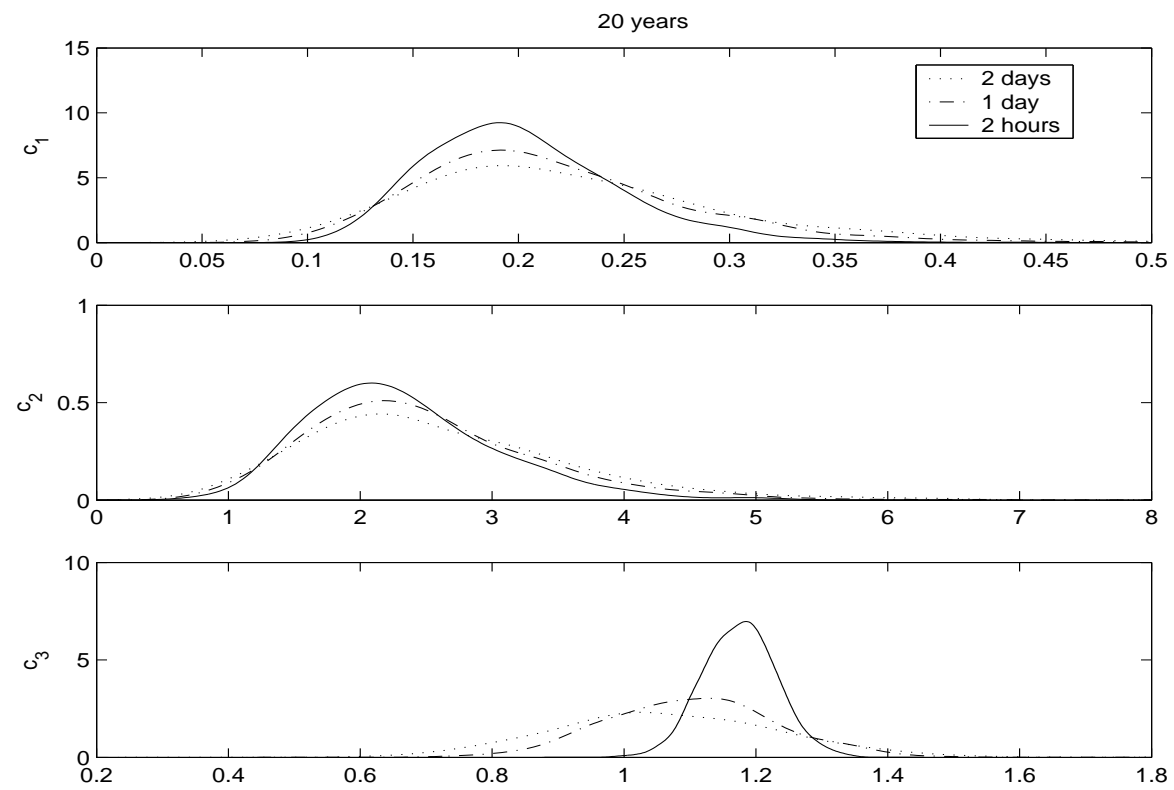

Figure 3: Density estimates of the continuous time parameters $c_{1}=0.18, c_{2}=2$ and $c_{3}=1.2$ of the GARCH diffusion model (1)-(2) inferred by the parameter estimates of the discre time GARCH-M model (17) at two-daily, daily and two-hourly sampling frequencies. Sample size 20 years.
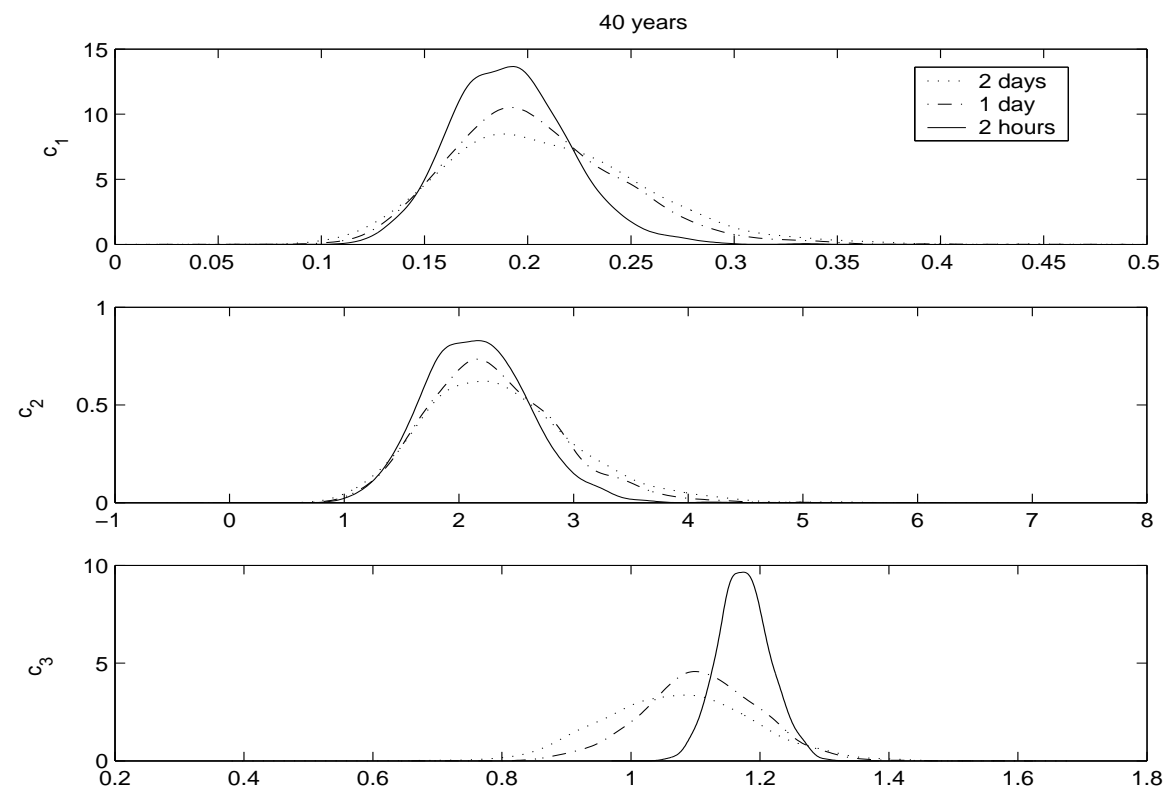

Figure 4: Density estimates of the continuous time parameters $c_{1}=0.18, c_{2}=2$ and $c_{3}=1.2$ of the GARCH diffusion model (1)-(2) inferred by the parameter estimates of the discre time GARCH-M model (17) at two-daily, daily and two-hourly sampling frequencies. Sample size 40 years. 


\section{References}

[1] Andersen T.G., L. Benzoni and J. Lund (2002), "An empirical investigation of continuoustime equity retur models", Journal of Finance, 57, 1239-1284.

[2] Ball C. A. and A. Roma (1994), "Stochastic Volatility Option Pricing", Journal of Financial and Quantitative Analysis, 29, 589-607.

[3] Black F. and M. Scholes (1973), "The Pricing of Options and Corporate Liabilities", Journal of Political Economy, 81, 637-659.

[4] Bodurtha J. and G. Courtadon (1987), "Tests of the American Option Pricing Model in the Foreign Currency Option Market", Journal of Financial and Quantitative Analysis, 22, $153-167$.

[5] Bollerslev T. and H. Zhou (2002), "Estimating Stochastic Volatility Diffusion using Conditional Moments of Integrated Volatility", Journal of Econometrics, 109, 33-65.

[6] Boyle P., M. Broadie and P. Glasserman (1997), "Monte Carlo Methods for Security Pricing", Journal of Economic Dynamics and Control, 21, 1267-1321.

[7] Chernov M. and E. Ghysels (2000), "A Study Towards a Unified Approach to the Joint Estimation of Objective and Risk-Neutral Measures for the Purpose of Options Valuation", Journal of Financial Economics, 56, 407-458.

[8] Chesney M. and L.O. Scott (1989), "Pricing European Currency Options: a Comparison of the Modified Black-Scholes Model and a Random Variance Model", Journal of Financial and Quantitative Analysis, 24, 267-284.

[9] Cox D.R. and H.D. Miller (1972), The Theory of Stochastic Processes, Chapman and Hall, London.

[10] Engle R.F. and T. Bollerslev (1986), "Modelling the Persistence of Conditional Variances", Econometric Reviews, 5, 1-50.

[11] Engle R.F. and G.G.J. Lee (1996), "Estimating Diffusion Models of Stochastic Volatility", in Rossi P.E. (Ed.), Modeling Stock Market Volatility: Bridging the Gap to Continuous Time, New York: Academic Press, 333-384.

[12] Fama E. (1965), "The Behaviour of Stock Prices", Journal of Business, 38, 34-105. 
[13] Fouque J., G. Papanicolaou and K.R. Sircar (2000), Derivatives in Financial Markets with Stochastic Volatility, Cambridge University Press, Cambridge.

[14] Garcia R., M.A. Lewis and E. Renault (2001), "Estimation of Objective and Risk-Neutral Distributions Based on Moments of the Integrated Volatility", working paper, Cirano.

[15] Genon-Catalot V., T. Jeantheau and C. Laredo (2000), "Stochastic Volatility Models as Hidden Markov Models and Statistical Applications", Bernoulli, 6, 1051-1079.

[16] Gesser V. and P. Poncet (1997), "Volatility Patterns: Theory and Some Evidence from the Dollar-Mark Option Market", The Journal of Derivatives, 5, 46-65.

[17] Guo D. (1996), "The Predictive Power of Implied Stochastic Variance from Currency Options", Journal of Futures Markets, 16, 915-942.

[18] Guo D. (1998), "The Risk Premium of Volatility Implicit in Currency Options", Journal of Business and Economic Statistics, 16, 498-507.

[19] Heston S. (1993), "A Closed-Form Solution for Options with Stochastic Volatility with Applications to Bond and Currency Options", Review of Financial Studies, 6, 327-343.

[20] Hull J. and A. White (1987), "The Pricing of Options on Assets with Stochastic Volatilities", Journal of Finance, 42, 281-300.

[21] Hull J. and A. White (1987a), "Hedging the Risks from Writing Foreign Currency Options", Journal of International money and Finance, 42, 131-152.

[22] Hull J. and A. White (1988), "An Analysis of the Bias in Option Pricing Caused by a Stochastic Volatility", Journal of International Economics, 24, 129-145.

[23] Karatzas I. and S. Shreve (1991), Brownian Motion and Stochastic Calculus, SpringerVerlag, New York

[24] Kloeden P.E. and E. Platen (1999), Numerical Solution of Stochastic Differential Equations, Springer-Verlag, New York.

[25] Jorion P. (1995), "Predicting Volatility in the Foreign Exchange Market", Journal of Finance, 50, 507-528.

[26] Jones C.S. (2003), "The Dynamics of Stochastic Volatility: Evidence from Underlying and Options Markets", Journal of Econometrics, 116, 181-224. 
[27] Lewis A.L. (2000), Option Valuation under Stochastic Volatility, Finance Press, California, USA.

[28] Lewis A.L. (2000a), "Analytical Expressions for the Moments of the Integrated Volatility in Affine Stochastic Volatility Models", working paper, Cirano.

[29] Mandelbrot B. (1963), "The Variation of Certain Speculative Prices", Journal of Business, 36, 394-419.

[30] Melenberg B. and B.J.M. Werker (2001), "The Pricing of Volatility Risk: An Empirical Example", working paper, Tilburg University.

[31] Melino A. and S.M. Turnbull (1990), "Pricing Foreign Currency Options with Stochastic Volatility", Journal of Econometrics, 45, 239-265.

[32] Merton R.C. (1973), "Theory of Rational Option Pricing", Bell Journal of Economics and Management Science, 4, 141-183.

[33] Nelson D.B. (1990), "ARCH Models as Diffusion Approximations", Journal of Econometrics, 45, 7-38.

[34] Renault E. and N. Touzi (1996), "Option Hedging and Implied Volatilities in a Stochastic Volatility Model", Mathematical Finance, 6, 279-302.

[35] Sircar K.R. and G.C. Papanicolaou (1999), "Stochastic Volatility, Smile and Asymptotics", Applied Mathematical Finance, 6, 107-145.

[36] Scott L.O. (1987), "Option Pricing when the Variance Change Randomly: Theory, Estimation and an Application", Journal of Financial and Quantitative Analysis, 22, 419-438.

[37] Taylor S.J., (1994), "Modeling Stochastic Volatility : a Review and Comparative Study", Mathematical Finance 4, 183-204.

[38] Taylor S.J. and X. Xu (1994), "The Magnitude of Implied Volatility Smiles: Theory and Empirical Evidence for Exchange Rates", The Review of Futures Markets, 13, 355-380.

[39] Wiggins J. (1987), "Option Values under Stochastic Volatility", Journal of Financial Economics, 19, 351-372.

[40] Wong E. (1964), "The Construction of a Class of Stationary Markoff Processes", in Belleman R. (Ed.), Sixteenth Symposium in Applied Mathematics, Stochastic Processes in Mathematical Physics and Engineering, American Mathematical Society, Providence, 264-276. 
[41] Xu X. and S.J. Taylor (1994), "Term Structure of Volatility Implied by Foreign Exchange Options", Journal of Finance and Quantitative Analysis, 29, 57-74. 